\title{
Synthesis, characterization, synergistic antimicrobial properties and molecular docking of sugar modified uridine derivatives
}

\author{
Jannatul MAOWA, ${ }^{1}$ Asraful ALAM, ${ }^{1}$ Kazi M. RANA, ${ }^{1}$ Sujan DEY,${ }^{2}$ Anowar HOSEN,${ }^{3}$ Yuki \\ FUJII, ${ }^{4}$ Imtiaj HASAN,${ }^{5}$ Yasuhiro OZEKI, ${ }^{6}$ and Sarkar M.A. KAWSAR ${ }^{* 1}$ \\ ${ }^{1}$ Department of Chemistry, Faculty of Science, University of Chittagong, Chittagong-4331, Bangladesh \\ ${ }^{2}$ Department of Microbiology, Faculty of Biological Science, University of Chittagong, Chittagong-4331, Bangladesh \\ ${ }^{3}$ Centre for Advanced Research in Sciences, University of Dhaka, Dhaka-1000, Bangladesh \\ ${ }^{4}$ Department of Functional Morphology, Graduate School of Pharmaceutical Sciences, Nagasaki International \\ University, 2825-7 Huis Ten Bosch, Sasebo, Nagasaki 859-3298, Japan \\ ${ }^{5}$ Department of Biochemistry and Molecular Biology, Faculty of Science, Rajshahi University, Rajshahi-6205, \\ Bangladesh \\ ${ }^{6}$ Department of Life and Environmental System Science, School of Sciences, Yokohama City University, 22-2 Seto, \\ Kanazawa-ku, Yokohama 236-0027, Japan
}

\begin{abstract}
Nucleosides and their analogues are an important, well-established class of clinically useful medicinal agents that exhibit antiviral and anticancer activity. Thus, our research group has focused on the synthesis of new nucleoside derivatives that could be tested for their broad-spectrum biological activity. In this study, two new series of nucleoside derivatives were synthesized from uridine (1) through facile two-step reactions using the direct acylation method, affording 5'-O-acyl uridine derivatives in good yields. The isolated uridine analogs were further transformed into two series of 2', $3^{\prime}$-di- $O$-acyl derivatives bearing a wide variety of functionalities in a single molecular framework to evaluate their antimicrobial activity. The new synthesized compounds were characterized through physicochemical, elemental and spectroscopic analysis, and all were screened for their in vitro antimicrobial activity against selected human and plant pathogenic strains. The test compounds revealed moderate to good antibacterial and antifungal activities and were more effective against fungal phytopathogens than against bacterial strains, while many of them exhibited better antimicrobial activity than standard antibiotics. Minimum inhibition concentration (MIC) and minimum bactericidal concentration (MBC) tests against all microorganisms were also conducted for five compounds based on their activity $(\mathbf{6}, \mathbf{1 1}, \mathbf{1 3}, \mathbf{1 6}$, and 17). In addition, all the derivatives were optimized using density functional theory (DFT) B3LYP/6-31g+(d,p) calculations to elucidate their thermal and molecular orbital properties. A molecular docking study was performed using the human protein 5WS1 to predict their binding affinity and modes, and ADMET and SwissADME calculations confirmed the improved pharmacokinetic properties of the compounds. Besides, structure-activity relationship (SAR), thermogravimetric analysis (TGA), and X-ray diffraction (XRD) studies were also performed. Thus, the improvement of the bioactivity of these compounds is expected to significantly contribute to the design of more antimicrobial agents for therapeutic use in the future.
\end{abstract}

Keywords: ADMET; antimicrobial; molecular docking; synthesis; characterization; uridine.

\section{Introduction}

Nucleosides can be found in all cells and are derived from the heterocyclic compounds purine and pyrimidine, which are also known as bases due to their basic nature. Cellular DNA and RNA include five major bases, which are linked to carbohydrates (ribose) in the cells, forming nucleosides. In particular, the purine and pyrimidine bases can couple either to D-ribose through a $\beta$ - $N$-glycosidic bond, which is developed between the anomeric carbon (C-1') of the ribose and the $N^{9}$-position of the purine or the $N^{1}$-position of the pyrimidine ring [1]. Both purine and pyrimidine nucleosides and their relative chemistry have contributed substantially to a better understanding of the biological processes at the molecular level [2, 3]. Moreover, nucleosides and their analogues are an established class of clinically useful medicinal agents with proven antiviral [4-7] and anticancer [8-10] activity. Nevertheless, they should be further investigated as antibacterial agents, since some derivatives have already shown moderate to good activity against specific bacterial strains [11].

Uridine (1) (Figure 1) is a glycosylated pyrimidine analog consisting of uracil attached to a ribose ring. Uridine, $\mathbf{1}$ is essential for the synthesis of RNA and biomembranes, while it is significantly involved in the regulation of normal physiological processes and the preparation of phospholipids in the brain. The concentrations of uridine in plasma, seminal fluid, and cerebrospinal fluid are high enough to be stored for use in cells [12].

Several efficient methods for selective acylation have been earlier reported using many acylating agents and varying reaction conditions $[13,14]$. In addition,

\footnotetext{
* Corresponding author. E-mail address: akawsarabe@yahoo.com (Sarkar M.A. Kawsar)
} 
various methods have been successfully developed and employed for the acylation of carbohydrates and nucleosides [15-17], among which the direct method is the most promising [17]. Moreover, a wide variety of acylated nucleoside derivatives have been synthesized and their biological activity has been evaluated [18-20]. More specifically, it was found that the combination of two or more acyl substituents in a single molecular framework significantly enhances the biological activity of their parent nuclei [21]. Furthermore, the benzene and substituted benzene nuclei play an important role in antimicrobial activity. It has also been observed that substituted nucleoside derivatives bearing aromatic, hetero-aromatic, and acyl substituents together with chlorine, bromine, and sulfur atoms can considerably improve the biological activity [22-24].

The main aim of the current work was to perform the selective 4-bromobenzoylation and palmitoylation (Scheme 1) of uridine (1) with 4-bromobenzoyl and palmitoyl chlorides, respectively, using the direct method. The obtained products ( 2 and 9 ) were further modified resulting in fifteen acylated derivatives and all the synthesized analogs were employed as test chemicals for antibacterial, antifungal, SAR, TGA, $\mathrm{XRD}$, and computational studies. Molecular docking was performed against the human protein gene 5WS1 to predict their binding affinity and binding modes [25, 26] for the first time.

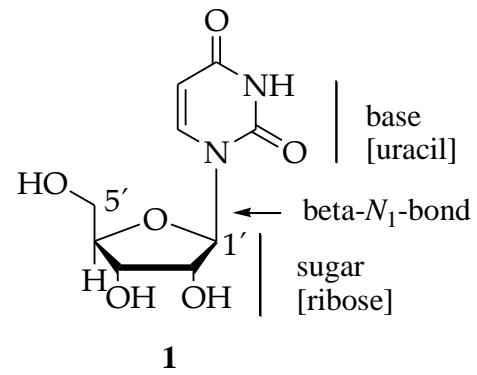

Figure 1. Chemical structure of uridine (1)

\section{Experimental}

\subsection{Materials and methods}

All chemicals were of analytical grade and used as received. The solvents were also of analytical grade and were purified using standard procedures. The infrared (IR) spectra were recorded using a Fourier-transform infrared (FTIR) spectrophotometer (Model-8900, Shimadzu, Japan) at a range of $200-4000 \mathrm{~cm}^{-1}$ at the Department of Chemistry, University of Chittagong, Bangladesh. Each reaction was monitored by collecting fractions at specific intervals and analyzed by thin-layer chromatography (TLC) (Kieselgel $\mathrm{GF}_{254}$ ) using a $\mathrm{CHCl}_{3} / \mathrm{CH}_{3} \mathrm{OH}$ mixture of different proportions. Column chromatography was performed with silica gel $\mathrm{G}_{60}$. The solvents were evaporated under reduced pressure using a VV-1 type vacuum rotary evaporator (Germany) with a bath temperature lower than $40{ }^{\circ} \mathrm{C}$. The ${ }^{1} \mathrm{H}$-NMR spectra were recorded in $\mathrm{CDCl}_{3}$ on a Brucker advance DPX $400 \mathrm{MHz}$ using tetramethylsilane (TMS) as internal standard ( $\delta$ in ppm) at Wazed Miah Science Research Centre (WMSRC), Jahangirnagar
University, Bangladesh. X-ray diffractometers consist of three basic elements: a X-ray tube, a sample holder, and a X-ray detector, and use $\mathrm{Cu} \mathrm{K}_{\alpha}$ or Mo $\mathrm{K}_{\alpha}$ radiations. For typical powder patterns, the data are collected at angles $(2 \theta)$ of $2^{\circ}-70^{\circ}$ that are present in the X-ray scan. In this study, the XRD analysis of the complexes was performed using an XRD-53 analyzer, Rigaku, Japan. The melting points were determined on an electrothermal melting point apparatus (Thermo Fisher Scientific, England) and were not corrected.

\subsection{Synthesis}

Our laboratory has already synthesized nucleoside derivatives bearing various acyl groups to explore their antimicrobial properties. Our current research involves the synthesis of uridine derivatives as test chemicals for antibacterial, antifungal, structure-activity relationship (SAR), and computational studies.

2.2.1. Synthesis of 4-bromobenzoyl uridine 2. A solution of uridine (1) $(200 \mathrm{mg}, 0.82 \mathrm{mmol})$ in anhydrous pyridine (Py, $3 \mathrm{~mL}$ ) was cooled to $0{ }^{\circ} \mathrm{C}$ and 4bromobenzoyl chloride (194 mg, 1.1 eq.) was added. The reaction mixture was continuously stirred at $0{ }^{\circ} \mathrm{C}$ for $6 \mathrm{~h}$ and then at room temperature (rt) overnight. The progress of the reaction was monitored by TLC $\left(\mathrm{CHCl}_{3} / \mathrm{CH}_{3} \mathrm{OH} 16: 1, \mathrm{v} / \mathrm{v}\right)$, which indicated the full conversion of the starting material into a single product $\left(R_{f}=0.52\right)$. The solution was poured into ice water with constant stirring, followed by extraction with chloroform $(3 \times 10 \mathrm{~mL})$. The combined chloroform layers were washed with dilute hydrochloric acid, a saturated aqueous sodium hydrogen carbonate solution, and distilled water. The organic layer was dried $\left(\mathrm{Na}_{2} \mathrm{SO}_{4}\right)$, filtered, and concentrated. Purification by column chromatography with $\left(\mathrm{CHCl}_{3} / n-\mathrm{C}_{6} \mathrm{H}_{14}\right)(1: 16)$ afforded the 4-bromobenzoyl derivative 2 ( $80 \mathrm{mg}, 55 \%)$ as crystalline solid, M.p. $105-107{ }^{\circ} \mathrm{C}\left(\mathrm{CHCl}_{3} / n-\mathrm{C}_{6} \mathrm{H}_{14}\right)$. 5'-O-(4-Bromobenzoyl)uridine (2): White crystalline solid; M.p.: $105-107{ }^{\circ} \mathrm{C} ; \mathrm{R}_{\mathrm{f}}=0.52\left(\mathrm{CHCl}_{3} / n-\mathrm{C}_{6} \mathrm{H}_{14}\right)=$ 16/1, v/v); yield: $56.22 \%$. FTIR (KBr) $v_{\max } / \mathrm{cm}^{-1} 3408$ 3550 (br, $-\mathrm{OH}), 1735$ (C=O). ${ }^{1} \mathrm{H}-\mathrm{NMR}(400 \mathrm{MHz}$, $\left.\mathrm{CDCl}_{3}\right), \delta$ ppm, $8.8(1 \mathrm{H}, \mathrm{s},-\mathrm{NH}), 7.93(2 \mathrm{H}, \mathrm{m}, \mathrm{Ar}-\mathrm{H})$, $7.83(2 \mathrm{H}, \mathrm{m}, \mathrm{Ar}-\mathrm{H}), 7.62(1 \mathrm{H}, \mathrm{d}, \mathrm{J}=7.8 \mathrm{~Hz}, \mathrm{H}-6), 6.19$ $\left(1 \mathrm{H}, \mathrm{d}, \mathrm{J}=5.6 \mathrm{~Hz}, \mathrm{H}-1^{\prime}\right), 6.0\left(1 \mathrm{H}, \mathrm{s}, 2^{\prime}-\mathrm{OH}\right), 5.87(1 \mathrm{H}$, $\mathrm{dd}, \mathrm{J}=2.0$ and $\left.12.0 \mathrm{~Hz}, \mathrm{H}-5^{\prime} \mathrm{a}\right), 5.77(1 \mathrm{H}, \mathrm{dd}, \mathrm{J}=2.1$ and $\left.12.1 \mathrm{~Hz}, \mathrm{H}-5^{\prime} \mathrm{b}\right), 5.62(1 \mathrm{H}, \mathrm{d}, \mathrm{J}=8.1 \mathrm{~Hz}, \mathrm{H}-5), 5.60$ $\left(1 \mathrm{H}, \mathrm{s}, 3^{\prime}-\mathrm{OH}\right), 4.72\left(1 \mathrm{H}, \mathrm{dd}, \mathrm{J}=2.0\right.$ and $\left.5.2 \mathrm{~Hz}, \mathrm{H}-4^{\prime}\right)$, $4.20\left(1 \mathrm{H}, \mathrm{d}, \mathrm{J}=5.4 \mathrm{~Hz}, \mathrm{H}-2^{\prime}\right), 4.15(1 \mathrm{H}, \mathrm{dd}, \mathrm{J}=7.2$ and $\left.5.3 \mathrm{~Hz}, \mathrm{H}-3^{\prime}\right)$. MS (m/z): $427.12[\mathrm{M}+1]^{+}$. Anal. Calcd. (\%) for $\mathrm{C}_{16} \mathrm{H}_{15} \mathrm{O}_{7} \mathrm{~N}_{2} \mathrm{Br}$ (426.12): C, 45.07; $\mathrm{H}, 3.52$. Found: C, 45.09; H, 3.50.

2.2.2. General procedure for the synthesis of derivatives 3-8. A solution of 2 (121 mg, $0.28 \mathrm{mmol})$ in dry pyridine $(\mathrm{Py}, 3 \mathrm{~mL})$ at $0{ }^{\circ} \mathrm{C}$ was treated with acetic anhydride $(0.08 \mathrm{~mL}, 4.0$ eq. $)$ and the mixture was stirred at $0{ }^{\circ} \mathrm{C}$ for $6-8 \mathrm{~h}$ followed by addition of catalytic amount of 4 dimethylaminopyridine (DMAP). A few pieces of ice was added to the reaction mixture to decompose unreacted (excess) acyl halide and extracted with chloroform $\left(\mathrm{CHCl}_{3}, 3 \times 5 \mathrm{~mL}\right)$. The $\mathrm{CHCl}_{3}$ layer was washed successively with $5 \%$ hydrochloric acid, saturated aqueous sodium hydrogen carbonate solution 
and brine. The $\mathrm{CHCl}_{3}$ layer was dried and concentrated under reduced pressure. The residue thus obtained on column chromatography $\left(\mathrm{CHCl}_{3} / \mathrm{CH}_{3} \mathrm{OH} 16: 1\right.$, v/v) afforded the acetyl derivative $\mathbf{3}(68.5 \mathrm{mg}, 46.89 \%)$ as crystalline solid. Recrystallization from a $\mathrm{CHCl}_{3} / \mathrm{CH}_{3} \mathrm{OH}$ mixture afforded 3 in the form of needles. M.p. $109-110{ }^{\circ} \mathrm{C}$. Following the same process, compounds 4 (82.6 mg), 5 (78.1 mg), 6 (56.4 mg), 7 $(112.5 \mathrm{mg})$, and $\mathbf{8}(90.2 \mathrm{mg})$ were successfully isolated. 2',3'-Di- $O$-acetyl-5'-O-(4-bromobenzoyl)uridine (3): White crystalline solid; M.p.: $109-111{ }^{\circ} \mathrm{C} ; \mathrm{R}_{\mathrm{f}}=0.51$ $\left(\mathrm{CHCl}_{3} / \mathrm{CH}_{3} \mathrm{OH}=16 / 1\right.$, v/v $)$; yield: 46.89\%. FTIR $(\mathrm{KBr}) v_{\max } / \mathrm{cm}^{-1} 1732,1740(\mathrm{C}=\mathrm{O}) .{ }^{1} \mathrm{H}-\mathrm{NMR} \quad(400$ $\left.\mathrm{MHz}, \mathrm{CDCl}_{3}\right), \delta \mathrm{ppm}, 8.81(1 \mathrm{H}, \mathrm{s},-\mathrm{NH}), 7.95(2 \mathrm{H}, \mathrm{m}$, $\mathrm{Ar}-\mathrm{H}), 7.81(2 \mathrm{H}, \mathrm{m}, \mathrm{Ar}-\mathrm{H}), 7.56(1 \mathrm{H}, \mathrm{d}, \mathrm{J}=7.8 \mathrm{~Hz}, \mathrm{H}-$ 6), 6.19 (1H, d, J = 5.8 Hz, H-1'), $5.88(1 \mathrm{H}, \mathrm{dd}, \mathrm{J}=2.1$ and $\left.12.0 \mathrm{~Hz}, \mathrm{H}-5^{\prime} \mathrm{a}\right), 5.77(1 \mathrm{H}, \mathrm{dd}, \mathrm{J}=2.2$ and $12.2 \mathrm{~Hz}$, $\left.\mathrm{H}-5^{\prime} \mathrm{b}\right), 5.70(1 \mathrm{H}, \mathrm{d}, \mathrm{J}=8.2 \mathrm{~Hz}, \mathrm{H}-5), 4.85(1 \mathrm{H}, \mathrm{d}, \mathrm{J}=$ $\left.5.2 \mathrm{~Hz}, \mathrm{H}-2^{\prime}\right), 4.73\left(1 \mathrm{H}, \mathrm{dd}, \mathrm{J}=7.4\right.$ and $\left.5.4 \mathrm{~Hz}, \mathrm{H}-3^{\prime}\right)$, $4.65\left(1 \mathrm{H}, \mathrm{m}, \mathrm{H}-4^{\prime}\right), 2.17,2.08(2 \times 3 \mathrm{H}, 2 \times \mathrm{s}, 2 \times$ $\left.\mathrm{CH}_{3} \mathrm{CO}-\right)$. MS (m/z): $502.04[\mathrm{M}+1]^{+}$. Anal. Calcd. (\%) for $\mathrm{C}_{20} \mathrm{H}_{19} \mathrm{O}_{9} \mathrm{~N}_{2} \mathrm{Br}$ (501.05): C, 47.90; H, 3.79. Found: C, 47.93; H, 3.82.

5'-O-(4-Bromobenzoyl)-2',3'-di- $O$-propionyluridine (4): Crystalline solid; M.p.: $112-114{ }^{\circ} \mathrm{C} ; \mathrm{R}_{\mathrm{f}}=0.52$ $\left(\mathrm{CHCl}_{3} / \mathrm{CH}_{3} \mathrm{OH}=16 / 1\right.$, v/v); yield: 64.22\%. FTIR (KBr) $v_{\max } / \mathrm{cm}^{-1} 1722(\mathrm{C}=\mathrm{O}) .{ }^{1} \mathrm{H}-\mathrm{NMR}(400 \mathrm{MHz}$, $\left.\mathrm{CDCl}_{3}\right), \delta$ ppm, $8.91(1 \mathrm{H}, \mathrm{s},-\mathrm{NH}), 7.94(2 \mathrm{H}, \mathrm{m}, \mathrm{Ar}-\mathrm{H})$, $7.88(2 \mathrm{H}, \mathrm{m}, \mathrm{Ar}-\mathrm{H}), 7.61(1 \mathrm{H}, \mathrm{d}, \mathrm{J}=7.6 \mathrm{~Hz}, \mathrm{H}-6), 6.10$ $\left(1 \mathrm{H}, \mathrm{d}, \mathrm{J}=5.4 \mathrm{~Hz}, \mathrm{H}-1^{\prime}\right), 5.88(1 \mathrm{H}, \mathrm{dd}, \mathrm{J}=2.1$ and 12.1 $\left.\mathrm{Hz}, \mathrm{H}-5^{\prime} \mathrm{a}\right), 5.70\left(1 \mathrm{H}, \mathrm{dd}, \mathrm{J}=2.0\right.$ and $\left.12.1 \mathrm{~Hz}, \mathrm{H}-5^{\prime} \mathrm{b}\right)$, $5.60(1 \mathrm{H}, \mathrm{d}, \mathrm{J}=7.6 \mathrm{~Hz}, \mathrm{H}-5), 4.81(1 \mathrm{H}, \mathrm{d}, \mathrm{J}=5.1 \mathrm{~Hz}$, $\left.\mathrm{H}-2^{\prime}\right), 4.53\left(1 \mathrm{H}, \mathrm{dd}, \mathrm{J}=7.2\right.$ and $\left.5.2 \mathrm{~Hz} \mathrm{H}-3^{\prime}\right), 4.30(1 \mathrm{H}$, m, H-4'), $2.42\left\{4 \mathrm{H}, \mathrm{m}, 2 \times \mathrm{CH}_{3} \mathrm{CH}_{2} \mathrm{CO}-\right\}, 1.21\{6 \mathrm{H}, \mathrm{m}$, $\left.2 \times \mathrm{CH}_{3} \mathrm{CH}_{2} \mathrm{CO}-\right\}$. MS (m/z): $539.02[\mathrm{M}+1]^{+}$. Anal. Calcd. (\%) for $\mathrm{C}_{22} \mathrm{H}_{23} \mathrm{O}_{9} \mathrm{~N}_{2} \mathrm{Br}$ (538.08): C, 49.07; $\mathrm{H}$, 4.28. Found: $\mathrm{C}, 49.04 ; \mathrm{H}, 4.30$.

5'-O-(4-Bromobenzoyl)-2',3'-di- $O$-butyryluridine (5): Light yelloe crystalline solid; M.p.: $114-117{ }^{\circ} \mathrm{C} ; \mathrm{R}_{\mathrm{f}}=$ $0.53\left(\mathrm{CHCl}_{3} / \mathrm{CH}_{3} \mathrm{OH}=16 / 1\right.$, v/v); yield: $46.34 \%$. FTIR (KBr) $v_{\max } / \mathrm{cm}^{-1} 1716(\mathrm{C}=\mathrm{O}) .{ }^{1} \mathrm{H}-\mathrm{NMR}(400 \mathrm{MHz}$, $\left.\mathrm{CDCl}_{3}\right), \delta \mathrm{ppm}, 8.51(1 \mathrm{H}, \mathrm{s},-\mathrm{NH}), 7.92(2 \mathrm{H}, \mathrm{m}, \mathrm{Ar}-\mathrm{H})$, $7.81(2 \mathrm{H}, \mathrm{m}, \mathrm{Ar}-\mathrm{H}), 7.63(1 \mathrm{H}, \mathrm{d}, \mathrm{J}=7.8 \mathrm{~Hz}, \mathrm{H}-6), 6.18$ $\left(1 \mathrm{H}, \mathrm{d}, \mathrm{J}=5.5 \mathrm{~Hz}, \mathrm{H}-1^{\prime}\right), 5.87(1 \mathrm{H}, \mathrm{dd}, \mathrm{J}=2.0$ and 12.0 $\left.\mathrm{Hz}, \mathrm{H}-5^{\prime} \mathrm{a}\right), 5.73\left(1 \mathrm{H}, \mathrm{dd}, \mathrm{J}=2.1\right.$ and $\left.12.2 \mathrm{~Hz}, \mathrm{H}-5^{\prime} \mathrm{b}\right)$, $5.58(1 \mathrm{H}, \mathrm{d}, \mathrm{J}=7.8 \mathrm{~Hz}, \mathrm{H}-5), 4.82(1 \mathrm{H}, \mathrm{d}, \mathrm{J}=5.3 \mathrm{~Hz}$, $\left.\mathrm{H}-2^{\prime}\right), 4.63\left(1 \mathrm{H}, \mathrm{dd}, \mathrm{J}=7.4\right.$ and $\left.5.5 \mathrm{~Hz} \mathrm{H}-3^{\prime}\right), 4.54(1 \mathrm{H}$, m, H-4'), $2.34\left\{4 \mathrm{H}, \mathrm{m}, 2 \times \mathrm{CH}_{3} \mathrm{CH}_{2} \mathrm{CH}_{2} \mathrm{CO}-\right\}, 1.65$ $\left(4 \mathrm{H}, \mathrm{m}, 2 \times \mathrm{CH}_{3} \mathrm{CH}_{2} \mathrm{CH}_{2} \mathrm{CO}-\right), 0.91\{6 \mathrm{H}, \mathrm{m}, 2 \times$ $\left.\mathrm{CH}_{3}\left(\mathrm{CH}_{2}\right)_{2} \mathrm{CO}-\right\}$. MS (m/z): $567.19[\mathrm{M}+1]^{+}$. Anal. Calcd. (\%) for $\mathrm{C}_{24} \mathrm{H}_{27} \mathrm{O}_{9} \mathrm{~N}_{2} \mathrm{Br}$ (566.21): C, 50.88; $\mathrm{H}$, 4.77. Found: C, 50.90; H, 4.74.

5'-O-(4-Bromobenzoyl)-2',3'-di- $O$-myristoyluridine

(6): White crystalline solid; M.p.: $85-88{ }^{\circ} \mathrm{C} ; \mathrm{R}_{\mathrm{f}}=0.55$ $\left(\mathrm{CHCl}_{3} / \mathrm{CH}_{3} \mathrm{OH}=16 / 1\right.$, v/v); yield: $88.05 \%$. FTIR (KBr) $v_{\max } / \mathrm{cm}^{-1} 1697(\mathrm{C}=\mathrm{O})$. ${ }^{1} \mathrm{H}-\mathrm{NMR}(400 \mathrm{MHz}$, $\left.\mathrm{CDCl}_{3}\right), \delta \mathrm{ppm}, 8.89(1 \mathrm{H}, \mathrm{s},-\mathrm{NH}), 7.91(2 \mathrm{H}, \mathrm{m}, \mathrm{Ar}-\mathrm{H})$, $7.83(2 \mathrm{H}, \mathrm{m}, \mathrm{Ar}-\mathrm{H}), 7.06(1 \mathrm{H}, \mathrm{d}, \mathrm{J}=7.5 \mathrm{~Hz}, \mathrm{H}-6), 6.10$ $\left(1 \mathrm{H}, \mathrm{d}, \mathrm{J}=5.5 \mathrm{~Hz}, \mathrm{H}-1^{\prime}\right), 5.63(1 \mathrm{H}, \mathrm{dd}, \mathrm{J}=2.0$ and 12.0 $\left.\mathrm{Hz}, \mathrm{H}-5^{\prime} \mathrm{a}\right), 5.17$ (1H, dd, $\mathrm{J}=2.2$ and $\left.12.2 \mathrm{~Hz}, \mathrm{H}-5^{\prime} \mathrm{b}\right)$, $5.02(1 \mathrm{H}, \mathrm{d}, \mathrm{J}=8.1 \mathrm{~Hz}, \mathrm{H}-5), 4.92(1 \mathrm{H}, \mathrm{d}, \mathrm{J}=5.2 \mathrm{~Hz}$, $\left.\mathrm{H}-2^{\prime}\right), 4.56\left(1 \mathrm{H}, \mathrm{dd}, \mathrm{J}=7.5\right.$ and $\left.5.5 \mathrm{~Hz}, \mathrm{H}-3^{\prime}\right), 4.65$
(1H,m, H-4'), $2.36\left\{4 \mathrm{H}, \mathrm{m}, 2 \times \mathrm{CH}_{3}\left(\mathrm{CH}_{2}\right)_{11} \mathrm{CH}_{2} \mathrm{CO}-\right\}$, $1.65\left\{4 \mathrm{H}, \mathrm{m}, 2 \times \mathrm{CH}_{3}\left(\mathrm{CH}_{2}\right)_{10} \mathrm{CH}_{2} \mathrm{CH}_{2} \mathrm{CO}-\right\}, 1.28\{40 \mathrm{H}$, $\left.\mathrm{m}, 2 \times \mathrm{CH}_{3}\left(\mathrm{CH}_{2}\right)_{10} \mathrm{CH}_{2} \mathrm{CH}_{2} \mathrm{CO}-\right\}, 0.93\{6 \mathrm{H}, \mathrm{m}, 2 \times$ $\left.\mathrm{CH}_{3}\left(\mathrm{CH}_{2}\right)_{12} \mathrm{CO}-\right\}$. MS (m/z): $612.16[\mathrm{M}+1]^{+}$. Anal. Calcd. (\%) for $\mathrm{C}_{29} \mathrm{H}_{21} \mathrm{O}_{9} \mathrm{~N}_{2} \mathrm{Br}$ (611.14): C, 56.96; $\mathrm{H}$, 3.44. Found: C, 56.99; H, 3.46.

5'-O-(4-Bromobenzoyl)-2',3'-di- $O$-trityluridine (7): Crystalline solid; M.p.: $\quad 85-87{ }^{\circ} \mathrm{C} ; \quad \mathrm{R}_{\mathrm{f}}=0.54$ $\left(\mathrm{CHCl}_{3} / \mathrm{CH}_{3} \mathrm{OH}=16 / 1\right.$, v/v); yield: $93.22 \%$. FTIR (KBr) $v_{\max } / \mathrm{cm}^{-1} 1723(\mathrm{C}=\mathrm{O}) .{ }^{1} \mathrm{H}-\mathrm{NMR}(400 \mathrm{MHz}$, $\left.\mathrm{CDCl}_{3}\right), \delta$ ppm, $8.88(1 \mathrm{H}, \mathrm{s},-\mathrm{NH}), 7.67(1 \mathrm{H}, \mathrm{d}, \mathrm{J}=7.4$ $\mathrm{Hz}, \mathrm{H}-6), 7.90$ (2H, m, Ar-H), 7.83 (2H, m, Ar-H), 7.65 $(2 \times 6 \mathrm{H}, \mathrm{m}, \mathrm{Ar}-\mathrm{H}), 7.31(2 \times 9 \mathrm{H}, \mathrm{m}, \mathrm{Ar}-\mathrm{H}), 6.29(1 \mathrm{H}, \mathrm{d}, \mathrm{J}$ $\left.=5.6 \mathrm{~Hz}, \mathrm{H}-1^{\prime}\right), 5.75(1 \mathrm{H}, \mathrm{dd}, \mathrm{J}=2.1$ and $11.8 \mathrm{~Hz}, \mathrm{H}-$ $\left.5^{\prime} \mathrm{a}\right), 5.45\left(1 \mathrm{H}, \mathrm{dd}, \mathrm{J}=2.0\right.$ and $\left.11.9 \mathrm{~Hz}, \mathrm{H}-5^{\prime} \mathrm{b}\right), 5.41($ $1 \mathrm{H}, \mathrm{d}, \mathrm{J}=8.2 \mathrm{~Hz}, \mathrm{H}-5), 5.0\left(1 \mathrm{H}, \mathrm{m}, \mathrm{H}-2^{\prime}\right), 4.67$ (1H, m, H-3'), 4.22 (1H, m, H-4'). MS (m/z): $911.10[\mathrm{M}+1]^{+}$. Anal. Calcd. (\%) for $\mathrm{C}_{54} \mathrm{H}_{43} \mathrm{O}_{7} \mathrm{~N}_{2} \mathrm{Br}$ (910.04): C, 71.21; $\mathrm{H}, 4.72$. Found: $\mathrm{C}, 71.23 ; \mathrm{H}, 4.70$.

2',3'-Di-O-(3-bromobenzoyl)-5'-O-(4-

bromobenzoyl)uridine (8): Light white crystalline solid; M.p.: $93-95^{\circ} \mathrm{C} ; \mathrm{R}_{\mathrm{f}}=0.55\left(\mathrm{CHCl}_{3} / \mathrm{CH}_{3} \mathrm{OH}=15 / 1\right.$, v/v); yield: $41.50 \%$. FTIR (KBr) $v_{\max } / \mathrm{cm}^{-1} 1746,1682$ $(\mathrm{C}=\mathrm{O}) .{ }^{1} \mathrm{H}-\mathrm{NMR}\left(400 \mathrm{MHz}, \mathrm{CDCl}_{3}\right), \delta \mathrm{ppm}, 9.1(1 \mathrm{H}, \mathrm{s}$, $-\mathrm{NH}), 7.99$ (1H, d, J = 7.2 Hz, H-6), $7.94(2 \mathrm{H}, \mathrm{m}, \mathrm{Ar}-$ H), 7.92 ( 2H, d, J = 7.6 Hz, Ar-H), $7.90(2 \mathrm{H}, \mathrm{s}, \mathrm{Ar}-\mathrm{H})$, $7.88(2 \mathrm{H}, \mathrm{m}, \mathrm{Ar}-\mathrm{H}), 7.78(2 \mathrm{H}, \mathrm{d}, \mathrm{J}=7.5 \mathrm{~Hz}), 7.47(2 \mathrm{H}$, $\mathrm{t}, \mathrm{J}=7.5 \mathrm{~Hz}), 6.49\left(1 \mathrm{H}, \mathrm{d}, \mathrm{J}=5.5 \mathrm{~Hz}, \mathrm{H}-1^{\prime}\right), 6.15(1 \mathrm{H}$, $\mathrm{dd}, \mathrm{J}=2.1$ and $\left.12.1 \mathrm{~Hz}, \mathrm{H}-5^{\prime} \mathrm{a}\right), 5.25(1 \mathrm{H}, \mathrm{dd}, \mathrm{J}=2.1$ and $12.0 \mathrm{~Hz}, \mathrm{H}-5^{\prime} \mathrm{b}$ ), 5.41 ( $\left.1 \mathrm{H}, \mathrm{d}, \mathrm{J}=8.2 \mathrm{~Hz}, \mathrm{H}-5\right), 4.92$ $\left(\left(1 \mathrm{H}, \mathrm{d}, \mathrm{J}=5.2 \mathrm{~Hz}, \mathrm{H}-2^{\prime}\right), 4.57(1 \mathrm{H}, \mathrm{dd}, \mathrm{J}=7.6\right.$ and 5.5 $\left.\mathrm{Hz}, \mathrm{H}-3^{\prime}\right), 4.21\left(1 \mathrm{H}, \mathrm{m}, \mathrm{H}-4^{\prime}\right) . \mathrm{MS}(\mathrm{m} / \mathrm{z}): 727.31$ $[\mathrm{M}+1]^{+}$. Anal. Calcd. (\%) for $\mathrm{C}_{34} \mathrm{H}_{67} \mathrm{O}_{9} \mathrm{~N}_{2} \mathrm{Br}$ (726.29): C, 56.20; H, 9.23. Found: C, 56.23; H, 9.21.

2.2.3. Synthesis of myristoyl Uuridine (9) and its derivatives (10-17). A solution of uridine (1) $(200 \mathrm{mg}$, $0.82 \mathrm{mmol})$ in dry (Py $(3 \mathrm{~mL})$ was cooled to $0{ }^{\circ} \mathrm{C}$ and myristoyl chloride $(0.24 \mathrm{~mL}, 1.1$ eq. $)$ was added. The mixture was stirred at $0{ }^{\circ} \mathrm{C}$ for $6 \mathrm{~h}$, and then was allowed to stand at rt overnight. The same workup as above was applied and the subsequent chromatographic purification with $\mathrm{CHCl}_{3} / \mathrm{CH}_{3} \mathrm{OH}$ afforded the myristoyl derivative 9 (225.0 $\mathrm{mg})$ as crystalline solid, which was used in the next step without further purification. The same synthesis and purification process as in section 3.2.2 was used to synthesize compounds $\mathbf{1 0}$ (110.12 mg), 11 (168.24 mg), 12 (340.19 mg), $\mathbf{1 3}$ (400.11 mg), 14 (203.21 mg), 15 (340.10 mg), 16 (450.21 mg), and 17 (181.16 mg).

5'-O-Myristoyluridine (9): Crystalline solid; M.p.: 100 $103{ }^{\circ} \mathrm{C} ; \mathrm{R}_{\mathrm{f}}=0.50\left(\mathrm{CHCl}_{3} / \mathrm{CH}_{3} \mathrm{OH}=16 / 1\right.$, v/v); yield: $82.33 \%$. FTIR (KBr) $v_{\max } / \mathrm{cm}^{-1} 3415-3488$ (br, $-\mathrm{OH}$ ), $1693(\mathrm{C}=\mathrm{O}) .{ }^{1} \mathrm{H}-\mathrm{NMR}\left(400 \mathrm{MHz}, \mathrm{CDCl}_{3}\right), \delta \mathrm{ppm}, 8.87$ $(1 \mathrm{H}, \mathrm{s},-\mathrm{NH}), 7.56(1 \mathrm{H}, \mathrm{d}, \mathrm{J}=7.6 \mathrm{~Hz}, \mathrm{H}-6), 6.26(1 \mathrm{H}$, d, J = 5.6 Hz, H-1'), 6.10 (1H, s, 2'-OH), 5.93 (1H, dd, $\mathrm{J}=2.1$ and $\left.12.1 \mathrm{~Hz}, \mathrm{H}-5^{\prime} \mathrm{a}\right), 5.76(1 \mathrm{H}, \mathrm{dd}, \mathrm{J}=2.1$ and $\left.12.2 \mathrm{~Hz}, \mathrm{H}-5^{\prime} \mathrm{b}\right), 5.71(1 \mathrm{H}, \mathrm{d}, \mathrm{J}=8.2 \mathrm{~Hz}, \mathrm{H}-5), 5.30$ $\left(1 \mathrm{H}, \mathrm{s}, 3^{\prime}-\mathrm{OH}\right), 4.75\left(1 \mathrm{H}, \mathrm{dd}, \mathrm{J}=2.3\right.$ and $\left.5.6 \mathrm{~Hz}, \mathrm{H}-4^{\prime}\right)$, $4.25\left(1 \mathrm{H}, \mathrm{d}, \mathrm{J}=5.8 \mathrm{~Hz}, \mathrm{H}-2^{\prime}\right), 4.10(1 \mathrm{H}, \mathrm{dd}, \mathrm{J}=7.4$ and $\left.5.4 \mathrm{~Hz}, \mathrm{H}-3^{\prime}\right) .2 .35\left\{2 \mathrm{H}, \mathrm{m}, \mathrm{CH}_{3}\left(\mathrm{CH}_{2}\right)_{11} \mathrm{CH}_{2} \mathrm{CO}-\right\}, 1.65$ $\left\{2 \mathrm{H}, \mathrm{m}, \mathrm{CH}_{3}\left(\mathrm{CH}_{2}\right)_{10} \mathrm{CH}_{2} \mathrm{CH}_{2} \mathrm{CO}-\right\}, 1.28\{20 \mathrm{H}, \mathrm{m}$, $\left.\mathrm{CH}_{3}\left(\mathrm{CH}_{2}\right)_{10} \mathrm{CH}_{2} \mathrm{CH}_{2} \mathrm{CO}-\right\}, \quad 0.90 \quad\{3 \mathrm{H}, \quad \mathrm{m}$, 
$\left.\mathrm{CH}_{3}\left(\mathrm{CH}_{2}\right)_{12} \mathrm{CO}-\right\}$. MS (m/z): $455[\mathrm{M}+1]^{+}$. Anal. Calcd. (\%) for $\mathrm{C}_{23} \mathrm{H}_{38} \mathrm{O}_{7} \mathrm{~N}_{2}$ (454.02): C, 60.79; $\mathrm{H}, 8.37$. Found: $\mathrm{C}, 60.81 ; \mathrm{H}, 8.35$.

2',3'-Di- $O$-acetyl-5'- $O$-myristoyluridine (10): White crystalline solid; M.p.: $80-82{ }^{\circ} \mathrm{C} ; \quad \mathrm{R}_{\mathrm{f}}=0.51$ $\left(\mathrm{CHCl}_{3} / \mathrm{CH}_{3} \mathrm{OH}=15 / 1\right.$, v/v); yield: $71.35 \%$. FTIR (KBr) $v_{\max } / \mathrm{cm}^{-1} 1700 \quad(\mathrm{C}=\mathrm{O}) .{ }^{1} \mathrm{H}-\mathrm{NMR}(400 \mathrm{MHz}$, $\left.\mathrm{CDCl}_{3}\right), \delta \mathrm{ppm}, 8.81(1 \mathrm{H}, \mathrm{s},-\mathrm{NH}), 7.46(1 \mathrm{H}, \mathrm{d}, \mathrm{J}=7.8$ $\mathrm{Hz}, \mathrm{H}-6), 6.16\left(1 \mathrm{H}, \mathrm{d}, \mathrm{J}=5.4 \mathrm{~Hz}, \mathrm{H}-1^{\prime}\right), 5.76(1 \mathrm{H}, \mathrm{dd}$, $\mathrm{J}=2.1$ and $\left.12.1 \mathrm{~Hz}, \mathrm{H}-5^{\prime} \mathrm{a}\right), 5.65(1 \mathrm{H}, \mathrm{dd}, \mathrm{J}=2.1$ and $\left.12.1 \mathrm{~Hz}, \mathrm{H}-5^{\prime} \mathrm{b}\right), 5.72(1 \mathrm{H}, \mathrm{d}, \mathrm{J}=8.2 \mathrm{~Hz}, \mathrm{H}-5), 4.88$ $\left(1 \mathrm{H}, \mathrm{d}, \mathrm{J}=5.3 \mathrm{~Hz}, \mathrm{H}-2^{\prime}\right), 4.74(1 \mathrm{H}, \mathrm{dd}, \mathrm{J}=7.2$ and 5.6 $\left.\mathrm{Hz}, \mathrm{H}-3^{\prime}\right), 4.67$ (1H,m, H-4'), $2.32 \quad\{2 \mathrm{H}, \mathrm{m}$, $\left.\mathrm{CH}_{3}\left(\mathrm{CH}_{2}\right)_{11} \mathrm{CH}_{2} \mathrm{CO}-\right\}, 2.15,2.11(2 \times 3 \mathrm{H}, 2 \times \mathrm{s}$, $\left.2 \times \mathrm{CH}_{3} \mathrm{CO}-\right), 1.62\left\{2 \mathrm{H}, \mathrm{m}, \mathrm{CH}_{3}\left(\mathrm{CH}_{2}\right)_{10} \mathrm{CH}_{2} \mathrm{CH}_{2} \mathrm{CO}-\right\}$, $1.26\left\{20 \mathrm{H}, \mathrm{m}, \mathrm{CH}_{3}\left(\mathrm{CH}_{2}\right){ }_{10} \mathrm{CH}_{2} \mathrm{CH}_{2} \mathrm{CO}-\right\}, 0.91\{3 \mathrm{H}, \mathrm{m}$, $\left.\mathrm{CH}_{3}\left(\mathrm{CH}_{2}\right)_{12} \mathrm{CO}-\right\}$. MS (m/z): $539.11[\mathrm{M}+1]^{+}$. Anal. Calcd. (\%) for $\mathrm{C}_{27} \mathrm{H}_{42} \mathrm{O}_{9} \mathrm{~N}_{2}$ (538.05): C, 60.22; H, 7.81. Found: C, 60.25; H, 7.78.

2',3'-Di- $O$-butyryl-5'- $O$-myristoyluridine (11): White crystalline solid; M.p.: $107-109{ }^{\circ} \mathrm{C} ; \quad \mathrm{R}_{\mathrm{f}}=0.52$ $\left(\mathrm{CHCl}_{3} / \mathrm{CH}_{3} \mathrm{OH}=16 / 1\right.$, v/v); yield: $66.03 \%$. FTIR (KBr) $v_{\max } / \mathrm{cm}^{-1} 1708(\mathrm{C}=\mathrm{O}) .{ }^{1} \mathrm{H}-\mathrm{NMR}(400 \mathrm{MHz}$, $\left.\mathrm{CDCl}_{3}\right), \delta$ ppm, $8.92(1 \mathrm{H}, \mathrm{s},-\mathrm{NH}), 7.74(1 \mathrm{H}, \mathrm{d}, \mathrm{J}=7.6$ $\mathrm{Hz}, \mathrm{H}-6), 6.38\left(1 \mathrm{H}, \mathrm{d}, \mathrm{J}=5.6 \mathrm{~Hz}, \mathrm{H}-1^{\prime}\right), 5.91(1 \mathrm{H}, \mathrm{dd}$, $\mathrm{J}=2.1$ and $\left.12.1 \mathrm{~Hz}, \mathrm{H}-5^{\prime} \mathrm{a}\right), 5.72(1 \mathrm{H}, \mathrm{dd}, \mathrm{J}=2.1$ and $\left.12.1 \mathrm{~Hz}, \mathrm{H}-5^{\prime} \mathrm{b}\right), 5.70(1 \mathrm{H}, \mathrm{d}, \mathrm{J}=7.7 \mathrm{~Hz}, \mathrm{H}-5), 5.53$ $\left(1 \mathrm{H}, \mathrm{d}, \mathrm{J}=5.2 \mathrm{~Hz}, \mathrm{H}-2^{\prime}\right), 4.71(1 \mathrm{H}, \mathrm{dd}, \mathrm{J}=7.6$ and 5.6 $\left.\mathrm{Hz} \mathrm{H}-3^{\prime}\right), 4.50$ (1H, m, H-4'), $2.88\{4 \mathrm{H}, \mathrm{m}, 2 \times$ $\left.\mathrm{CH}_{3} \mathrm{CH}_{2} \mathrm{CH}_{2} \mathrm{CO}-\right\}, 2.34\left\{2 \mathrm{H}, \mathrm{m}, \mathrm{CH}_{3}\left(\mathrm{CH}_{2}\right)_{11} \mathrm{CH}_{2} \mathrm{CO}-\right.$ \}, $1.76\left(4 \mathrm{H}, \mathrm{m}, 2 \times \mathrm{CH}_{3} \mathrm{CH}_{2} \mathrm{CH}_{2} \mathrm{CO}-\right), 1.65\{2 \mathrm{H}, \mathrm{m}$, $\left.\mathrm{CH}_{3}\left(\mathrm{CH}_{2}\right)_{10} \mathrm{CH}_{2} \mathrm{CH}_{2} \mathrm{CO}-\right\}, \quad 1.27 \quad\{20 \mathrm{H}, \quad \mathrm{m}$, $\left.\mathrm{CH}_{3}\left(\mathrm{CH}_{2}\right)_{10} \mathrm{CH}_{2} \mathrm{CH}_{2} \mathrm{CO}-\right\}, \quad 1.06\{6 \mathrm{H}, \quad \mathrm{m}, 2 \times$ $\left.\mathrm{CH}_{3}\left(\mathrm{CH}_{2}\right)_{2} \mathrm{CO}-\right\}, 0.96\left\{3 \mathrm{H}, \mathrm{m}, \mathrm{CH}_{3}\left(\mathrm{CH}_{2}\right)_{12} \mathrm{CO}-\right\} . \mathrm{MS}$ $(\mathrm{m} / \mathrm{z}): 595.22[\mathrm{M}+1]^{+}$. Anal. Calcd. $(\%)$ for $\mathrm{C}_{31} \mathrm{H}_{50} \mathrm{O}_{9} \mathrm{~N}_{2}$ (594.19): C, 62.63; H, 8.42. Found: C, 62.65; H, 8.45.

5'-O-Myristoyl-2',3'-di- $O$-octanoyluridine

(12): Crystalline solid; M.p.: 97-99 ${ }^{\circ} \mathrm{C} ; \quad \mathrm{R}_{\mathrm{f}}=0.50$ $\left(\mathrm{CHCl}_{3} / \mathrm{CH}_{3} \mathrm{OH}=15 / 1\right.$, v/v); yield: $63.11 \%$. FTIR (KBr) $v_{\max } / \mathrm{cm}^{-1} 1702 \sim 1725$ (br) $(\mathrm{C}=\mathrm{O}) .{ }^{1} \mathrm{H}-\mathrm{NMR}(400$ $\left.\mathrm{MHz}, \mathrm{CDCl}_{3}\right), \delta$ ppm, $8.92(1 \mathrm{H}, \mathrm{s},-\mathrm{NH}), 7.56(1 \mathrm{H}, \mathrm{d}, \mathrm{J}$ $=7.6 \mathrm{~Hz}, \mathrm{H}-6), 6.28\left(1 \mathrm{H}, \mathrm{d}, \mathrm{J}=5.6 \mathrm{~Hz}, \mathrm{H}-1^{\prime}\right), 6.12(1 \mathrm{H}$, m, H-5'a), 5.88 (1H, m, H-5’b), $5.72(1 \mathrm{H}, \mathrm{d}, \mathrm{J}=8.4$ $\mathrm{Hz}, \mathrm{H}-5), 5.53$ (1H, m, H-2’), 4.82 (1H, m, H-3'), 4.62 $\left(1 \mathrm{H}, \mathrm{m}, \mathrm{H}-4^{\prime}\right), 2.37\left\{2 \mathrm{H}, \mathrm{m}, \mathrm{CH}_{3}\left(\mathrm{CH}_{2}\right)_{11} \mathrm{CH}_{2} \mathrm{CO}-\right\}$, $2.35\left\{4 \mathrm{H}, \mathrm{m}, 2 \times \mathrm{CH}_{3}\left(\mathrm{CH}_{2}\right)_{5} \mathrm{CH}_{2} \mathrm{CO}-\right\}, 1.66\{2 \mathrm{H}, \mathrm{m}$, $\left.\mathrm{CH}_{3}\left(\mathrm{CH}_{2}\right)_{10} \mathrm{CH}_{2} \mathrm{CH}_{2} \mathrm{CO}-\right\}, \quad 1.64\{4 \mathrm{H}, \quad \mathrm{m}, 2 \times$ $\left.\mathrm{CH}_{3}\left(\mathrm{CH}_{2}\right)_{4} \mathrm{CH}_{2} \mathrm{CH}_{2} \mathrm{CO}-\right\}, \quad 1.29\{20 \mathrm{H}, \quad \mathrm{m}$, $\left.\mathrm{CH}_{3}\left(\mathrm{CH}_{2}\right)_{10} \mathrm{CH}_{2} \mathrm{CH}_{2} \mathrm{CO}-\right\}, \quad 1.25\{16 \mathrm{H}, \quad \mathrm{m}, 2 \times$ $\left.\mathrm{CH}_{3}\left(\mathrm{CH}_{2}\right)_{4}\left(\mathrm{CH}_{2}\right)_{2} \mathrm{CO}-\right\}, 0.98\left\{3 \mathrm{H}, \mathrm{m}, \mathrm{CH}_{3}\left(\mathrm{CH}_{2}\right)_{12} \mathrm{CO}-\right.$ \}, $0.96\left\{6 \mathrm{H}, \mathrm{m}, 2 \times \mathrm{CH}_{3}\left(\mathrm{CH}_{2}\right)_{6} \mathrm{CO}-\right\} . \mathrm{MS}(\mathrm{m} / \mathrm{z}): 707.06$ $[\mathrm{M}+1]^{+}$. Anal. Calcd. (\%) for $\mathrm{C}_{39} \mathrm{H}_{66} \mathrm{O}_{9} \mathrm{~N}_{2}$ (706.07): C, 66.29; H, 9.35. Found: C, 66.31; H, 9.37.

2',3'-Di- $O$-lauroyl-5'- $O$-myristoyluridine

(13):

Crystalline solid; M.p.: $102-104{ }^{\circ} \mathrm{C} ; \quad \mathrm{R}_{\mathrm{f}}=0.52$ $\left(\mathrm{CHCl}_{3} / \mathrm{CH}_{3} \mathrm{OH}=16 / 1\right.$, v/v); yield: $90.23 \%$. FTIR (KBr) $v_{\max } / \mathrm{cm}^{-1} 1704(\mathrm{C}=\mathrm{O})$. ${ }^{1} \mathrm{H}-\mathrm{NMR}(400 \mathrm{MHz}$, $\left.\mathrm{CDCl}_{3}\right), \delta \mathrm{ppm}, 8.92(1 \mathrm{H}, \mathrm{s},-\mathrm{NH}), 7.85(1 \mathrm{H}, \mathrm{d}, \mathrm{J}=7.6$ $\mathrm{Hz}, \mathrm{H}-6), 6.26\left(\left(1 \mathrm{H}, \mathrm{d}, \mathrm{J}=5.5 \mathrm{~Hz}, \mathrm{H}-1^{\prime}\right), 5.81(1 \mathrm{H}, \mathrm{m}\right.$, H-5'a), $5.73\left(1 \mathrm{H}, \mathrm{m}, \mathrm{H}-5^{\prime} \mathrm{b}\right), 5.61(1 \mathrm{H}, \mathrm{d}, \mathrm{J}=8.2 \mathrm{~Hz}$, $\mathrm{H}-5), 5.40\left(1 \mathrm{H}, \mathrm{d}, \mathrm{J}=5.5 \mathrm{~Hz}, \mathrm{H}-2^{\prime}\right), 4.83(1 \mathrm{H}, \mathrm{dd}, \mathrm{J}=$ 7.4 and $\left.5.5 \mathrm{~Hz}, \mathrm{H}-3^{\prime}\right), 4.60\left(1 \mathrm{H}, \mathrm{m}, \mathrm{H}-4^{\prime}\right), 2.33$ \{4H, m, $\begin{array}{lcccc}\left.2 \times \mathrm{CH}_{3}\left(\mathrm{CH}_{2}\right)_{9} \mathrm{CH}_{2} \mathrm{CO}-\right\}, & 2.32 \quad\{2 \mathrm{H}, & \mathrm{m}, \\ \left.\mathrm{CH}_{3}\left(\mathrm{CH}_{2}\right)_{11} \mathrm{CH}_{2} \mathrm{CO}-\right\}, & 1.62 & \{2 \mathrm{H}, & \mathrm{m}, \\ \left.\mathrm{CH}_{3}\left(\mathrm{CH}_{2}\right)_{10} \mathrm{CH}_{2} \mathrm{CH}_{2} \mathrm{CO}-\right\}, & 1.61 & \{4 \mathrm{H}, \quad \mathrm{m}, & 2 & \times \\ \left.\mathrm{CH}_{3}\left(\mathrm{CH}_{2}\right)_{8} \mathrm{CH}_{2} \mathrm{CH}_{2} \mathrm{CO}-\right\}, & 1.26 & \{32 \mathrm{H}, \quad \mathrm{m}, & 2 & \times \\ \left.\mathrm{CH}_{3}\left(\mathrm{CH}_{2}\right)_{8} \mathrm{CH}_{2} \mathrm{CH}_{2} \mathrm{CO}-\right\}, & 0.88 & \{6 \mathrm{H}, \quad \mathrm{m}, & 2 & \times \\ \left.\mathrm{CH}_{3}\left(\mathrm{CH}_{2}\right)_{10} \mathrm{CO}_{-}\right\}, & 1.27 & \{20 \mathrm{H}, & \mathrm{m}, \\ \left.\mathrm{CH}_{3}\left(\mathrm{CH}_{2}\right)_{10} \mathrm{CH}_{2} \mathrm{CH}_{2} \mathrm{CO}-\right\}, & 0.95 & \{3 \mathrm{H}, & \mathrm{m},\end{array}$ $\left.\mathrm{CH}_{3}\left(\mathrm{CH}_{2}\right)_{12} \mathrm{CO}-\right\}$. MS (m/z): $819.01[\mathrm{M}+1]^{+}$. Anal. Calcd. (\%) for $\mathrm{C}_{47} \mathrm{H}_{82} \mathrm{O}_{9} \mathrm{~N}_{2}$ (818.0): C, 68.95; H, 10.02 . Found: C, 68.93; H, 10.05.

5'-O-Myristoyl-2',3'-di- $O$-palmitoyluridine

White crystalline solid; M.p.: $84-86{ }^{\circ} \mathrm{C} ; \mathrm{R}_{\mathrm{f}}=0.50$ $\left(\mathrm{CHCl}_{3} / \mathrm{CH}_{3} \mathrm{OH}=15 / 1\right.$, v/v $)$; yield: $98.03 \%$. FTIR (KBr) $v_{\max } / \mathrm{cm}^{-1} 1704(\mathrm{C}=\mathrm{O}) .{ }^{1} \mathrm{H}-\mathrm{NMR}(400 \mathrm{MHz}$, $\left.\mathrm{CDCl}_{3}\right), \delta$ ppm, $8.89(1 \mathrm{H}, \mathrm{s},-\mathrm{NH}), 7.62(1 \mathrm{H}, \mathrm{d}, \mathrm{J}=7.2$ $\mathrm{Hz}, \mathrm{H}-6), 6.21\left(1 \mathrm{H}, \mathrm{d}, \mathrm{J}=5.6 \mathrm{~Hz}, \mathrm{H}-1^{\prime}\right), 6.01(1 \mathrm{H}$, dd, $\mathrm{J}=2.0$ and $\left.12.0 \mathrm{~Hz}, \mathrm{H}-5^{\prime} \mathrm{a}\right), 5.66(1 \mathrm{H}, \mathrm{dd}, \mathrm{J}=2.0$ and $\left.12.0 \mathrm{~Hz}, \mathrm{H}-5^{\prime} \mathrm{b}\right), 5.62(1 \mathrm{H}, \mathrm{d}, \mathrm{J}=8.1 \mathrm{~Hz}, \mathrm{H}-5), 5.51$ $\left(1 \mathrm{H}, \mathrm{d}, \mathrm{J}=5.5 \mathrm{~Hz}, \mathrm{H}-2^{\prime}\right), 5.48\left(1 \mathrm{H}, \mathrm{m}, \mathrm{H}-3^{\prime}\right), 4.63(1 \mathrm{H}$, m, H-4'), $2.34\left\{4 \mathrm{H}, \mathrm{m}, 2 \times \mathrm{CH}_{3}\left(\mathrm{CH}_{2}\right)_{13} \mathrm{CH}_{2} \mathrm{CO}-\right\}, 2.33$ $\left\{2 \mathrm{H}, \quad \mathrm{m}, \quad \mathrm{CH}_{3}\left(\mathrm{CH}_{2}\right)_{11} \mathrm{CH}_{2} \mathrm{CO}-\right\}, \quad 1.63\{2 \mathrm{H}, \mathrm{m}$, $\left.\mathrm{CH}_{3}\left(\mathrm{CH}_{2}\right)_{10} \mathrm{CH}_{2} \mathrm{CH}_{2} \mathrm{CO}-\right\}, \quad 1.26\{52 \mathrm{H}, \quad \mathrm{m}, \quad 2 \times$ $\left.\mathrm{CH}_{3}\left(\mathrm{CH}_{2}\right)_{13} \mathrm{CH}_{2} \mathrm{CO}-\right\}, \quad 1.24\{20 \mathrm{H}, \quad \mathrm{m}$, $\left.\mathrm{CH}_{3}\left(\mathrm{CH}_{2}\right)_{10} \mathrm{CH}_{2} \mathrm{CH}_{2} \mathrm{CO}-\right\}, \quad 0.92\{6 \mathrm{H}, \quad \mathrm{m}, \quad 2 \times$ $\left.\mathrm{CH}_{3}\left(\mathrm{CH}_{2}\right)_{14} \mathrm{CO}-\right\}, 0.90\left\{3 \mathrm{H}, \mathrm{m}, \mathrm{CH}_{3}\left(\mathrm{CH}_{2}\right)_{12} \mathrm{CO}-\right\} . \mathrm{MS}$ $(\mathrm{m} / \mathrm{z}): 931.08[\mathrm{M}+1]^{+}$. Anal. Calcd. $(\%)$ for $\mathrm{C}_{55} \mathrm{H}_{98} \mathrm{O}_{9} \mathrm{~N}_{2}$ (930.11): C, 70.97; H, 10.54. Found: C, 70.99; H, 10.50.

5'-O-Myristoyl-2',3'-di- $O$-stearoyluridine (15): White crystalline solid; M.p.: $111-113{ }^{\circ} \mathrm{C} ; \quad \mathrm{R}_{\mathrm{f}}=0.51$ $\left(\mathrm{CHCl}_{3} / \mathrm{CH}_{3} \mathrm{OH}=16 / 1\right.$, v/v); yield: $56.38 \%$. FTIR $(\mathrm{KBr}) v_{\max } / \mathrm{cm}^{-1} 1712(\mathrm{C}=\mathrm{O}) .{ }^{1} \mathrm{H}-\mathrm{NMR}(400 \mathrm{MHz}$, $\left.\mathrm{CDCl}_{3}\right), \delta$ ppm, $8.98(1 \mathrm{H}, \mathrm{s},-\mathrm{NH}), 7.58(1 \mathrm{H}, \mathrm{d}, \mathrm{J}=7.0$ $\mathrm{Hz}, \mathrm{H}-6), 6.12$ (1H, d, J = 5.5 Hz, H-1' $), 6.0$ (1H, dd, J $=2.1$ and $\left.12.1 \mathrm{~Hz}, \mathrm{H}-5^{\prime} \mathrm{a}\right), 5.58(1 \mathrm{H}, \mathrm{dd}, \mathrm{J}=2.1$ and 12.1 $\left.\mathrm{Hz}, \mathrm{H}-5^{\prime} \mathrm{b}\right), 5.56(1 \mathrm{H}, \mathrm{d}, \mathrm{J}=8.0 \mathrm{~Hz}, \mathrm{H}-5), 5.50(1 \mathrm{H}, \mathrm{d}$, $\left.\mathrm{J}=5.6 \mathrm{~Hz}, \mathrm{H}-2^{\prime}\right), 5.48\left(1 \mathrm{H}, \mathrm{m}, \mathrm{H}-3^{\prime}\right), 4.54(1 \mathrm{H}, \mathrm{m}, \mathrm{H}-$ $\left.4^{\prime}\right), 2.37\left\{4 \mathrm{H}, \mathrm{m}, 2 \times \mathrm{CH}_{3}\left(\mathrm{CH}_{2}\right)_{15} \mathrm{CH}_{2} \mathrm{CO}-\right\}, 2.34\{2 \mathrm{H}$, $\left.\mathrm{m}, \quad \mathrm{CH}_{3}\left(\mathrm{CH}_{2}\right)_{11} \mathrm{CH}_{2} \mathrm{CO}-\right\}, \quad 1.65 \quad\{2 \mathrm{H}, \quad \mathrm{m}$, $\left.\mathrm{CH}_{3}\left(\mathrm{CH}_{2}\right)_{10} \mathrm{CH}_{2} \mathrm{CH}_{2} \mathrm{CO}-\right\}, \quad 1.28 \quad\{2 \mathrm{H}, \quad \mathrm{m}$, $\left.\mathrm{CH}_{3}\left(\mathrm{CH}_{2}\right)_{10} \mathrm{CH}_{2} \mathrm{CH}_{2} \mathrm{CO}-\right\}, \quad 1.24 \quad\{60 \mathrm{H}, \mathrm{m}$, $\left.2 \times \mathrm{CH}_{3}\left(\mathrm{CH}_{2}\right)_{15} \mathrm{CH}_{2} \mathrm{CO}-\right\}, \quad 0.98 \quad\{6 \mathrm{H}, \quad \mathrm{m}, \quad 2 \times$ $\left.\mathrm{CH}_{3}\left(\mathrm{CH}_{2}\right)_{14} \mathrm{CO}-\right\}, 0.90\left\{3 \mathrm{H}, \mathrm{m}, \mathrm{CH}_{3}\left(\mathrm{CH}_{2}\right)_{12} \mathrm{CO}-\right\} . \mathrm{MS}$ $(\mathrm{m} / \mathrm{z}): 736.22[\mathrm{M}+1]^{+}$. Anal. Calcd. $(\%)$ for $\mathrm{C}_{41} \mathrm{H}_{71} \mathrm{O}_{9} \mathrm{~N}_{2}$ (735.21): C, 66.94; H, 9.66. Found: C, 66.96; H, 9.70.

5'-O-Myristoyl-2',3'-di- $O$-trityluridine

(16):

Crystalline solid; M.p.: $109-110{ }^{\circ} \mathrm{C} ; \quad \mathrm{R}_{\mathrm{f}}=0.50$ $\left(\mathrm{CHCl}_{3} / \mathrm{CH}_{3} \mathrm{OH}=15 / 1\right.$, v/v); yield: $96.19 \%$. FTIR (KBr) $v_{\max } / \mathrm{cm}^{-1} 1694(\mathrm{C}=\mathrm{O}) .{ }^{1} \mathrm{H}-\mathrm{NMR}$ (400 MHz, $\left.\mathrm{CDCl}_{3}\right), \delta \mathrm{ppm}, 8.94(1 \mathrm{H}, \mathrm{s},-\mathrm{NH}), 7.54(1 \mathrm{H}, \mathrm{d}, \mathrm{J}=7.1$ $\mathrm{Hz}, \mathrm{H}-6), 7.51(2 \times 6 \mathrm{H}, \mathrm{m}, \mathrm{Ar}-\mathrm{H}), 7.31(2 \times 9 \mathrm{H}, \mathrm{m}, \mathrm{Ar}-$ H), $6.10\left(1 \mathrm{H}, \mathrm{d}, \mathrm{J}=5.4 \mathrm{~Hz}, \mathrm{H}-1^{\prime}\right), 6.20(1 \mathrm{H}, \mathrm{dd}, \mathrm{J}=2.1$ and $\left.12.1 \mathrm{~Hz}, \mathrm{H}-5^{\prime} \mathrm{a}\right), 5.54(1 \mathrm{H}, \mathrm{dd}, \mathrm{J}=2.1$ and $12.1 \mathrm{~Hz}$, $\left.\mathrm{H}-5^{\prime} \mathrm{b}\right), 5.42(1 \mathrm{H}, \mathrm{d}, \mathrm{J}=8.0 \mathrm{~Hz}, \mathrm{H}-5), 5.35(1 \mathrm{H}, \mathrm{d}, \mathrm{J}=$ $\left.5.6 \mathrm{~Hz}, \mathrm{H}-2^{\prime}\right), 5.33\left(1 \mathrm{H}, \mathrm{m}, \mathrm{H}-3^{\prime}\right), 4.50$ (1H, m, H-4’), $2.34\left\{2 \mathrm{H}, \mathrm{m}, \mathrm{CH}_{3}\left(\mathrm{CH}_{2}\right)_{11} \mathrm{CH}_{2} \mathrm{CO}-\right\}, 1.64\{2 \mathrm{H}, \mathrm{m}$, $\left.\mathrm{CH}_{3}\left(\mathrm{CH}_{2}\right)_{10} \mathrm{CH}_{2} \mathrm{CH}_{2} \mathrm{CO}-\right\}, \quad 1.26 \quad\{20 \mathrm{H}, \quad \mathrm{m}$, $\left.\mathrm{CH}_{3}\left(\mathrm{CH}_{2}\right)_{10} \mathrm{CH}_{2} \mathrm{CH}_{2} \mathrm{CO}-\right\}, \quad 0.91 \quad\{3 \mathrm{H}, \quad \mathrm{m}$, $\left.\mathrm{CH}_{3}\left(\mathrm{CH}_{2}\right)_{12} \mathrm{CO}-\right\}$. MS (m/z): $939.03[\mathrm{M}+1]^{+}$. Anal. Calcd. (\%) for $\mathrm{C}_{61} \mathrm{H}_{66} \mathrm{O}_{7} \mathrm{~N}_{2}$ (938.0): C, 78.04; H, 7.04. Found: C, 78.06; H, 7.07.

2',3'-Di- $O$-(3-bromobenzoyl)-5'- $O$-myristoyluridine (17): Crystalline solid; M.p. point: $110-112{ }^{\circ} \mathrm{C} ; \mathrm{R}_{\mathrm{f}}=$ 
$0.54\left(\mathrm{CHCl}_{3} / \mathrm{CH}_{3} \mathrm{OH}=16 / 1, \mathrm{v} / \mathrm{v}\right)$; yield: $68.41 \%$. FTIR (KBr) $v_{\max } / \mathrm{cm}^{-1} 1725(\mathrm{C}=\mathrm{O}) .{ }^{1} \mathrm{H}-\mathrm{NMR}(400 \mathrm{MHz}$, $\left.\mathrm{CDCl}_{3}\right), \delta \mathrm{ppm}, 8.88(1 \mathrm{H}, \mathrm{s},-\mathrm{NH}), 7.84(2 \mathrm{H}, \mathrm{d}, \mathrm{J}=7.5$ $\mathrm{Hz}, \mathrm{Ar}-\mathrm{H}), 7.58(2 \mathrm{H}, \mathrm{s}, \mathrm{Ar}-\mathrm{H}), 7.53(1 \mathrm{H}, \mathrm{d}, \mathrm{J}=7.5 \mathrm{~Hz}$, $\mathrm{H}-6), 7.46(2 \mathrm{H}, \mathrm{d}, \mathrm{J}=7.6 \mathrm{~Hz}, \mathrm{Ar}-\mathrm{H}), 7.38(2 \mathrm{H}, \mathrm{t}, \mathrm{J}=$ $7.6 \mathrm{~Hz}, \mathrm{Ar}-\mathrm{H}), 6.01\left(1 \mathrm{H}, \mathrm{d}, \mathrm{J}=6.5 \mathrm{~Hz}, \mathrm{H}-1^{\prime}\right), 5.89(1 \mathrm{H}$, m, H-5’a), $5.81\left(1 \mathrm{H}, \mathrm{m}, \mathrm{H}-5^{\prime} \mathrm{b}\right), 5.78(1 \mathrm{H}, \mathrm{d}, \mathrm{J}=7.8 \mathrm{~Hz}$, $\mathrm{H}-5), 5.18\left(1 \mathrm{H}, \mathrm{d}, \mathrm{J}=5.2 \mathrm{~Hz}, \mathrm{H}-2^{\prime}\right), 5.11(1 \mathrm{H}, \mathrm{dd}, \mathrm{J}=$ 7.6 and $\left.5.5 \mathrm{~Hz}, \mathrm{H}-3^{\prime}\right), 4.30\left(1 \mathrm{H}, \mathrm{m}, \mathrm{H}-4^{\prime}\right), 2.32\{2 \mathrm{H}$, $\left.\mathrm{m}, \quad \mathrm{CH}_{3}\left(\mathrm{CH}_{2}\right)_{11} \mathrm{CH}_{2} \mathrm{CO}-\right\}, \quad 1.62\{2 \mathrm{H}, \quad \mathrm{m}$, $\left.\mathrm{CH}_{3}\left(\mathrm{CH}_{2}\right)_{10} \mathrm{CH}_{2} \mathrm{CH}_{2} \mathrm{CO}-\right\}, \quad 1.24 \quad\{2 \mathrm{H}, \quad \mathrm{m}$, $\left.\mathrm{CH}_{3}\left(\mathrm{CH}_{2}\right)_{10} \mathrm{CH}_{2} \mathrm{CH}_{2} \mathrm{CO}-\right\}, \quad 0.89 \quad\{3 \mathrm{H}, \quad \mathrm{m}$, $\left.\mathrm{CH}_{3}\left(\mathrm{CH}_{2}\right)_{12} \mathrm{CO}-\right\}$. MS (m/z): $821.15[\mathrm{M}+1]^{+}$. Anal. Calcd. (\%) for $\mathrm{C}_{37} \mathrm{H}_{46} \mathrm{O}_{9} \mathrm{~N}_{2} \mathrm{Br}_{2}$ (820.11): C, 54.15; H, 5.61. Found: C, 54.17; H, 5.63.

\subsection{Antimicrobial and antifungal screening studies}

The synthesized test compounds (Scheme 1 and 2) were subjected to antibacterial and antifungal screening studies against two Gram-positive and three Gramnegative bacterial strains viz., Bacillus subtilis ATCC 6633, Staphylococcus aureus ATCC 6538, Escherichia coli ATCC 8739, Salmonella abony NCTC 6017, and Pseudomonas aeruginosa ATCC 9027; two phytopathogenic fungi viz., Aspergillus niger ATCC 16404, and Aspergillus flavus ATCC 204304. The test tube cultures of the pathogens were obtained from the Department of Microbiology, University of Chittagong, Bangladesh.

\subsection{Screening of antibacterial activity}

The disk diffusion method [27] was used to check in vitro the sensitivity of bacteria to the synthesized compounds. A Mueller Hinton agar medium was distributed in sterilized Petri dishes, followed by the addition of a bacterial suspension $(0.1 \mathrm{~mL})$ and about $15-20 \mathrm{~mL}$ of the agar medium. Paper disks $(5 \mathrm{~mm}$ in diameter), soaked with the test chemicals ( $20 \mu \mathrm{L} / \mathrm{disk})$, were used for antibacterial analysis. For the sensitivity spectrum analysis, the agar medium plates were uniformly selected with the test organisms, and the disks were prepared with a given amount of the test chemicals. A disk containing each solvent system was used as the experimental control (C). The plates were kept at $4{ }^{\circ} \mathrm{C}$ for 2 to $4 \mathrm{~h}$ for the maximum diffusion of the compounds. During this time, the dried disks absorbed water from the surrounding media. The test materials were then placed into the solution and diffused in the media according to the natural laws of molecular diffusion through agar gels. The plates were then incubated at $37^{\circ} \mathrm{C}$ for $24 \mathrm{~h}$ in an inverted position to allow the maximum bacterial growth. After incubation, the easily observed zones of inhibition (i.e., distinct zones surrounding the disks that contained no microbial growth) were detected and measured. The diameter of the transparent scale included the diameter of the disks and that of each experiment, and the measurements were performed in triplicate. All the results were compared with the standard azithromycin antibiotic (Beximco Pharmaceuticals Ltd., Bangladesh).

\subsection{Determination of MIC and MBC}

The minimum inhibition concentration (MIC) and minimum bactericidal concentration $(M B C)$ of the compounds that showed activity against the aforementioned organisms were determined using the broth microdilution method [28] by applying different concentrations of the compounds to the same bacterial loads in a nutrient broth.

\subsection{Screening of mycelial growth}

The poisoned food technique [29] was employed to screen the antifungal activity in which using potato dextrose agar (PDA) as the culture medium. The test compounds were dissolved in dimethyl sulfoxide (DMSO) at a concentration of $1 \%(\mathrm{w} / \mathrm{v})$ and $0.1 \mathrm{~mL}$ of the solution (containing $1 \mathrm{mg}$ of the respective test compound) was transferred to a sterile Petri dish with a sterilized pipette. Afterward, $20 \mathrm{~mL}$ of the medium was poured into the Petri dish and allowed to solidify. The inoculation was then performed in the center of each Petri dish with a $5 \mathrm{~mm}$ mycelium block of each fungus. The mycelium block was prepared by applying a corkscrew to the growing area of a 5-day culture of the test fungi on PDA. The blocks were placed at the center of each Petri dish in an inverted position to maximize the contact between the mycelium and the culture medium. The inoculation plates were incubated at $25 \pm 2{ }^{\circ} \mathrm{C}$ and the experiment was conducted in triplicate. A control sample (i.e., PDA without test chemicals) was also maintained under the same conditions. After 5 days of incubation, the diameter of the fungal radial mycelial growth was measured. The average of three measurements was considered as the radial mycelia growth of the fungus in $\mathrm{mm}$.

\subsection{SAR study}

The SAR assays were performed following the membrane permeation concept disclosed by Kim and Hunt [30, 31].

\subsection{Thermogravimetric analysis}

A TGA-50H thermogravimetric analyzer (TGA-50H, SHIMADZU) was used to investigate the thermal stability and degradation of the synthesized compounds. For thermogravimetric analysis (TGA), a specific amount of the test compounds was sealed in an alumina pan and then heated from $\mathrm{rt}$ to $600{ }^{\circ} \mathrm{C}$ at a heating rate $10{ }^{\circ} \mathrm{C} / \mathrm{min}$ under a constant $\mathrm{N}_{2}$ flow rate $(10 \mathrm{~mL} / \mathrm{min})$.

\subsection{X-ray powder diffraction}

$\mathrm{X}$-ray powder diffraction (XRD) is a rapid analytical technique mainly used for phase identification of crystalline materials and can provide information on unit cell dimensions. The analyzed material is finely ground, homogenized, and its average bulk composition is determined.

\subsection{Optimization of uridine derivatives}

In computer-aided drug design, quantum mechanical methods are widely used to calculate pharmacokinetic properties [32]. Herein, all computations of uridine derivatives were carried out using the Gaussian 09W software package [32]. The Becke-Lee-Yang-Parr (BLYP) density functional theory (DFT) [33, 34] threeparameter hybrid model with a correlation functional under 3-21G basis set was employed to optimize and predict their thermal and molecular orbital properties. The dipole moment, enthalpy, free energy, and 
electrostatic potential were calculated for all the compounds. The features of the frontier highest occupied molecular orbital (HOMO) and lowest unoccupied molecular orbital (LUMO) were calculated at the same level of theory. For each uridine derivative, the HOMO-LUMO energy gap, hardness $(\eta)$, and softness $(S)$ were calculated from the energies of the frontier HOMO and LUMO, taking into account the Parr and Pearson interpretation of DFT and the Koopmans theorem [35)] on the correlation between the ionization potential $(I)$ and the electron affinities $(E)$ with HOMO and LUMO energy $(\varepsilon)$.

\subsection{Preparation of protein, molecular docking, and ADMET prediction}

Three uridine derivatives $(\mathbf{6}, \mathbf{1 1}$, and 16) were subjected to molecular docking studies against the human protein gene 5WS1. The crystal structure of 5WS1 was obtained from the protein data bank (PDB) database (PDB ID:5WS1) [36]. For docking, the water molecules were removed from the crystal structure, followed by the addition of non-polar hydrogen atoms using PyMol software packages (version 1.3) [37]. The energy minimization of the protein was implemented by the Swiss-Pdb viewer software (version 4.1.0) [38] and the optimized drugs were used for the molecular docking study against 5WS1. Finally, the molecular docking simulation was performed using the PyRx software (version 0.8) [39], where the protein was considered as the macromolecule and the drug as the ligand. In this analysis, rigid docking was performed, where all rotatable bonds were converted to non-rotatable with a center grid box size of $60.5798,64.8065,57.7209 \AA$ along the $\mathrm{x}, \mathrm{y}$, and $\mathrm{z}$ axes, respectively. The ADMET and SwissADME online databases were used to predict the pharmacokinetic and physicochemical properties [40].

\subsection{Statistical analysis}

For each investigated parameter, the experimental results were presented as mean \pm standard error for three replicates. Two-tailed Student's t-tests were used for statistical analysis. Only $p$ values lower than 0.05 were considered as statistically significant.

\section{Results and discussion}

\subsection{Selective 4-bromobenzoylation of uridine for the synthesis of analogue 2}

Usual workup and chromatographic purification furnished the 4-bromobenzoyl derivative 2 . Two characteristic peaks at 3408-3550 (broad) and 1735 $\mathrm{cm}^{-1}$ were identified in the FTIR spectrum of the uridine derivative (2), which were assigned to the $\mathrm{O}-\mathrm{H}$ and $\mathrm{C}=\mathrm{O}$ stretching, respectively. The formation of the monosubstitution product was revealed by its ${ }^{1} \mathrm{H}-\mathrm{NMR}$ spectrum, where one multiplet at $7.93 \mathrm{ppm}(\mathrm{ArH}, 2 \mathrm{H})$ and one multiplet at $7.83 \mathrm{ppm}(\mathrm{ArH}, 2 \mathrm{H})$ were observed, corresponding to the aromatic ring protons of the 4bromobenzoyl group in the molecule. Moreover, a considerable downfield shift to 5.87 (dd, $J=2.0,12.0$ $\mathrm{Hz}, 5$ 'a) and $5.77 \mathrm{ppm}\left(\mathrm{dd}, J=2.1,12.1 \mathrm{~Hz}, 5^{\prime} \mathrm{b}\right)$ was observed in the C-5' proton compared to the normal value [24], suggesting the introduction of the 4bromobenzoyl group at position 5'. The rest peaks of the ${ }^{1} \mathrm{H}-\mathrm{NMR}$ spectrum were successfully assigned to the structure of 2 .

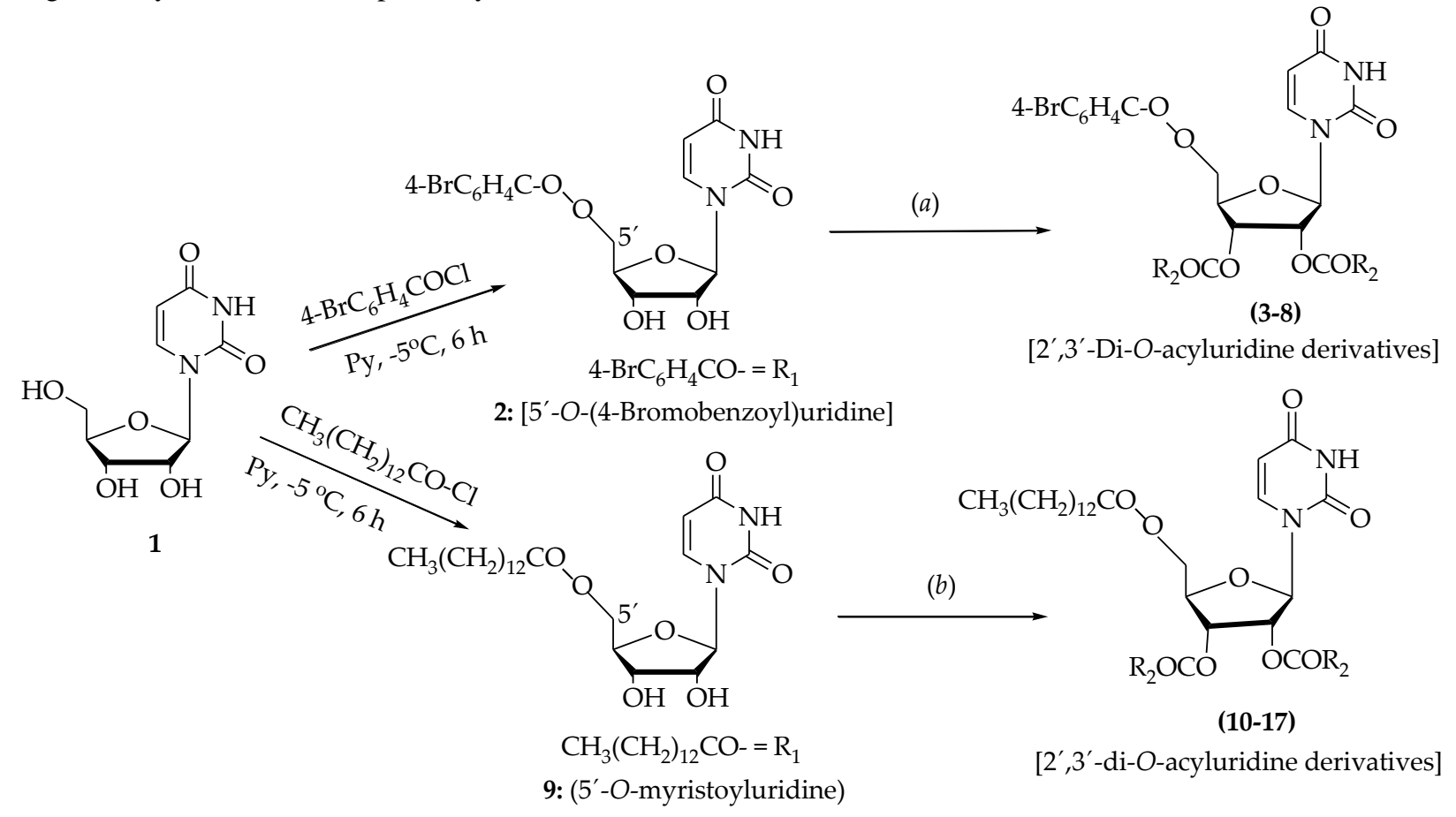

Scheme 1. Synthetic pathway of the compounds 2-17

Mechanistically, the formation of compound $\mathbf{2}$ may include the attachment of 4-bromobenzoyl chloride to the most reactive and less sterically hindered primary $\mathrm{OH}$ group of the ribose moiety at 5' position, forming the 5'-O-4-bromobenzoate (2) as the only product. Moreover, a molecular ion peak at $\mathrm{m} / \mathrm{z} 427.1271$ $[\mathrm{M}+\mathrm{H}]^{+}$was detected in the mass spectrum of compound 2, corresponding to the molecular formula 
$\mathrm{C}_{16} \mathrm{H}_{15} \mathrm{O}_{7} \mathrm{~N}_{2} \mathrm{Br}$. Therefore, all the spectroscopic data confirmed the formation of the 5'-O-(4bromobenzoyl)uridine structure (2), as shown in Scheme 1. Thus, the unimolecular 4-bromobenzoylation of uridine (1) at low temperature in dry Py was selective for the C-5' position, suggesting a reactivity of the uridine $\mathrm{OH}$ groups as 5'-OH > 2'-OH, 3'-OH.

\subsection{Synthesis of the 5'-O-(4-bromobenzoyl)uridine (2) derivatives 3-8}

Several derivatives of analogue 2 were also prepared to provide additional information for their structure and to isolate novel derivatives of synthetic and biological importance. The acylation mainly occurred on the two free $-\mathrm{OH}$ groups at $\mathrm{C}-2^{\prime}$ and $\mathrm{C}-3$ ', thus confirming the structure of the 5 '- $O$-(triphenylmethyl)uridine derivative (2). Its structure was further confirmed by the preparation of the di- $O$-acetyl derivative $\mathbf{3}$ using acetic anhydride in dry $\mathrm{Py}$ in the presence of 4dimethylaminopyridine (DMAP) as the catalyst, in $87.89 \%$ yield. In the FTIR spectrum of $\mathbf{3}$, two peaks were observed due to $\mathrm{C}=\mathrm{O}$ stretching at 1732 and $1740 \mathrm{~cm}^{-1}$. However, the absence of the peak that corresponded to the $\mathrm{OH}$ group(s) indicated the di- $O$ acetylation of the molecule. Two singlets at 2.17 and $2.08 \mathrm{ppm}(3 \mathrm{H})$ were detected in the ${ }^{1} \mathrm{H}-\mathrm{NMR}$ spectrum that could be assigned to the two acetyl methyl protons $\left(\mathrm{COCH}_{3}\right)$. Moreover, the $\mathrm{H}-2^{\prime}$ and $\mathrm{H}-3^{\prime}$ protons were detected at $4.85(\mathrm{~d}, J=5.2 \mathrm{~Hz})$ and $4.73 \mathrm{ppm}(\mathrm{dd}, J$ $=7.4,5.4 \mathrm{~Hz}$ ), respectively, and were slightly shifted compared to those of the precursor 5'-O-4bromobenzoate 2 at 4.20 and $4.15 \mathrm{ppm}$, respectively. These downfield shifts of $\mathrm{H}-2^{\prime}$ and $\mathrm{H}-3^{\prime}$ indicated the attachment of the acetyloxy groups at positions $\mathrm{C}-2^{\prime}$ and C-3', respectively. In addition, the mass spectrum exhibited a molecular ion peak at $\mathrm{m} / \mathrm{z} 502.0511[\mathrm{M}+\mathrm{H}]^{+}$, which corresponded to a molecular formula of $\mathrm{C}_{20} \mathrm{H}_{19} \mathrm{O}_{9} \mathrm{~N}_{2} \mathrm{Br}$. Thus, based on the above spectroscopic data, the structure of acetate $\mathbf{3}$ was determined to be 2',3'-di- $O$-acetyl-5'- $O$-(4-bromobenzoyl)uridine (Scheme 1).

Similarly, the di-O-acylation of compound 2 with five different acylating agents afforded the 2',3'-di- $O$ acylates 4-8 in good yields (Scheme 1). All these compounds were characterized by FTIR, ${ }^{1} \mathrm{H}-\mathrm{NMR}$, and mass spectrometry (MS). As in the case of analog $\mathbf{3}$, the $\mathrm{H}-2^{\prime}$ and $\mathrm{H}-3^{\prime}$ protons of 4-8 shifted downfield significantly compared to the corresponding peaks of compound 2.

\subsection{Selective myristoylation of uridine for the synthesis of derivatives 9}

5'-O-Myristoyluridine (9) was prepared as a white crystalline solid from uridine (1) in $82.33 \%$ yield upon treatment with myristoyl chloride in dry Py at $-5{ }^{\circ} \mathrm{C}$ (Scheme 1). The structure of the myristoyl derivative (9) was established by analyzing its elemental data, and the FTIR, ${ }^{1} \mathrm{H}-\mathrm{NMR}$, and MS spectra. In its ${ }^{1} \mathrm{H}$ NMR spectrum, two multiplets at $2.35\left(\mathrm{CH}_{3}\left(\mathrm{CH}_{2}\right)_{11} \mathrm{CH}_{2} \mathrm{CO}-\right.$, $2 \mathrm{H})$ and $1.65 \mathrm{ppm}\left(\mathrm{CH}_{3}\left(\mathrm{CH}_{2}\right)_{10} \mathrm{CH}_{2} \mathrm{CH}_{2} \mathrm{CO}-, 2 \mathrm{H}\right)$, a multiplet at $1.28 \mathrm{ppm}\left(\mathrm{CH}_{3}\left(\mathrm{CH}_{2}\right)_{10} \mathrm{CH}_{2} \mathrm{CH}_{2} \mathrm{CO}-, 20 \mathrm{H}\right)$, and a multiplet at $0.90 \mathrm{ppm}\left(\mathrm{CH}_{3}\left(\mathrm{CH}_{2}\right)_{12} \mathrm{CO}-, 3 \mathrm{H}\right)$ suggested the attachment of one myristoyl group to compound 9. The downfield shifts of the C-5' protons to $5.93(\mathrm{dd}, J=2.1,12.1 \mathrm{~Hz}, 5 ' \mathrm{a})$ and $5.76 \mathrm{ppm}(\mathrm{dd}$, $J=2.1,12.2 \mathrm{~Hz}, 5^{\prime} \mathrm{b}$ ) from the corresponding peaks of uridine and the efficient matching of the remaining protons with the rest of the peaks confirmed the substitution of C-5' by the myristoyl group. Moreover, a molecular ion peak was identified at $\mathrm{m} / \mathrm{z} 455.7197$ $[\mathrm{M}+\mathrm{H}]^{+}$, corresponding to the molecular formula $\mathrm{C}_{23} \mathrm{H}_{38} \mathrm{O}_{7} \mathrm{~N}_{2}$.

\subsection{Synthesis of the 5'-O-myristoyluridine derivatives 10-17}

The myristoate analog 9 was converted to a series of acetyl derivatives to further elucidate its structure (Scheme 1). In particular, the acetyl derivative $\mathbf{1 0}$ was prepared in $71.35 \%$ yield following the procedure reported in previous section. The FTIR, ${ }^{1} \mathrm{H}$ NMR, and MS data allowed the identification of its structure to 2',3'-di- $O$-acetyl-5'- $O$-myristoyluridine (10). Additional analogs, including the butyryloate $\mathbf{1 1}$, the trityloate $\mathbf{1 6}$, and the 3-bromobenzoate 17, were prepared to confirm the structure of 9. Their structures were easily established by analyzing their FTIR, ${ }^{1} \mathrm{H}-\mathrm{NMR}$, MS, elemental, and physicochemical properties. Analog 9 was also derivatized using four fatty acid chlorides, i.e., octanoyl, lauroyl, palmitoyl, and stearoyl chloride, and direct acylation methods.

The corresponding acyl derivatives $(\mathbf{1 2}, \mathbf{1 3}, \mathbf{1 4}$, and 15) were isolated in good yields and complete analysis of their FTIR, ${ }^{1} \mathrm{H}-\mathrm{NMR}$, and mass spectra together with other properties confirmed the formation of the corresponding 2',3'-di- $O$-substitution products. All the obtained products were then employed for antibacterial, antifungal, SAR, and computational studies.

\subsection{Antimicrobial evaluation of uridine derivatives}

The newly synthesized compounds (2-17) were screened for their antimicrobial activity against five human pathogenic bacteria and two phytopathogenic fungi, measured in terms of zone of inhibition in $\mathrm{mm}$. For a comparative study, the antimicrobial activity of two standard antibiotics, azithromycin and nystatin, were also evaluated against the same microorganisms.

\subsection{Determination of the antibacterial activity of uridine derivatives against bacteria}

The inhibition findings indicated that analogs 11 $(22 \pm 0.18 \mathrm{~mm})$ and $13(25 \pm 0.27 \mathrm{~mm})$ were more active against $B$. subtilis than azithromycin, while $\mathbf{1 6}$ was more active against $S$. aureus $(23 \pm 0.31 \mathrm{~mm})$ than the standard antibiotic (Table 1). Thus, significant inhibition was observed for compounds 6, 7, 10, and 12, whereas compounds $\mathbf{1}$ and $\mathbf{2}$ were inactive against both Gram-positive microorganisms.

Furthermore, based on the data presented in Table 1, compounds 2 and 9 showed the most extensive inhibition against the Gram-negative bacterium $E$. coli (14 \pm 0.24 and $14 \pm 0.18 \mathrm{~mm}$, respectively), while the rest analogs had moderate activity. Moreover, the greatest antibacterial screening inhibition against $S$. abony was exhibited by compound $\mathbf{1 7}$ with a zone of inhibition of $25 \pm 0.35 \mathrm{~mm}$, which was also quite higher than that of azithromycin, whereas compounds $\mathbf{8}$ and $\mathbf{1 4}$ exhibited lower inhibitory activity. In contrast, the antibacterial activity results against $P$. aeruginosa 
indicated compounds $\mathbf{3}, \mathbf{1 1}, \mathbf{1 3}$, and $\mathbf{1 6}$ as the most active. Furthermore, compounds 6, 9, 12, and 17 exhibited also moderate but lower activity than the previously mentioned analogs, while compound $\mathbf{1 0}$ exhibited good inhibition against $P$. aeruginosa, comparable to that of azithromycin. It should also be noted that the obtained inhibition results were in line with our previous research works [41, 42].

Table 1. Zone of inhibition of the synthesized compounds 1-17 against Gram-positive \& Gram-negative bacteria

\begin{tabular}{cccccc}
\hline \multirow{2}{*}{ Compd. } & \multicolumn{5}{c}{ Diameter of zone of inhibition (mm) } \\
\cline { 2 - 5 } & B. subtilis (+ve) & S. aureus (+ve) & E. coli (-ve) & S. abony (-ve) & P. aeruginosa (-ve) \\
\hline $\mathbf{2}$ & NI & NI & $* 14 \pm 0.24$ & NI & $12 \pm 0.16$ \\
$\mathbf{3}$ & NI & $10 \pm 0.14$ & $10 \pm 0.19$ & $12 \pm 0.15$ & $* 18 \pm 0.24$ \\
$\mathbf{4}$ & NI & $10 \pm 0.15$ & $10 \pm 0.21$ & $13 \pm 0.22$ & $10 \pm 0.19$ \\
$\mathbf{5}$ & $9 \pm 0.12$ & $14 \pm 0.20$ & $10 \pm 0.16$ & $10 \pm 0.16$ & NI \\
$\mathbf{6}$ & $13 \pm 0.14$ & $* 17 \pm 0.22$ & $12 \pm 0.21$ & $12 \pm 0.18$ & $15 \pm 0.19$ \\
$\mathbf{7}$ & $15 \pm 0.24$ & $14 \pm 0.17$ & $11 \pm 0.19$ & $15 \pm 0.21$ & $8 \pm 0.15$ \\
$\mathbf{8}$ & $10 \pm 0.16$ & $10 \pm 0.14$ & $7 \pm 0.11$ & $9 \pm 0.14$ & $11 \pm 0.18$ \\
$\mathbf{9}$ & NI & $9 \pm 0.14$ & $* 14 \pm 0.18$ & $15 \pm 0.24$ & $15 \pm 0.11$ \\
$\mathbf{1 0}$ & $* 18 \pm 0.25$ & $10 \pm 0.13$ & $10 \pm 0.17$ & NI & $* 17 \pm 0.19$ \\
$\mathbf{1 1}$ & $* 22 \pm 0.18$ & $* 17 \pm 0.15$ & $12 \pm 0.20$ & $12 \pm 0.18$ & $* 20 \pm 0.34$ \\
$\mathbf{1 2}$ & $10 \pm 0.16$ & $15 \pm 0.22$ & $11 \pm 0.18$ & NI & $15 \pm 0.23$ \\
$\mathbf{1 3}$ & $* 25 \pm 0.27$ & $15 \pm 0.19$ & $11 \pm 0.19$ & NI & $* 21 \pm 0.35$ \\
$\mathbf{1 4}$ & NI & $10 \pm 0.15$ & $10 \pm 0.15$ & $9 \pm 0.12$ & NI \\
$\mathbf{1 5}$ & NI & $14 \pm 0.21$ & $10 \pm 0.19$ & $10 \pm 0.14$ & $10 \pm 0.18$ \\
$\mathbf{1 6}$ & NI & $* 23 \pm 0.31$ & $7 \pm 0.10$ & $15 \pm 0.22$ & $* 21 \pm 0.30$ \\
17 & NI & $7 \pm 0.11$ & $7 \pm 0.11$ & $* 25 \pm 0.35$ & $15 \pm 0.18$ \\
Azithromycin & $* * 19 \pm 0.22$ & $* * 18 \pm 0.21$ & $E$. coli & $* * 19 \pm 0.31$ & $* * 17 \pm 0.27$ \\
\hline
\end{tabular}

The data are presented as mean \pm SD and the values are represented for triplicate experiments. Statistically significant inhibition $(p<0.05)$ is marked with an asterisk $(*)$ for test compounds and a double asterisk $(* *)$ for the reference antibiotic azithromycin. NI = No inhibition; $(+\mathrm{ve})=\mathrm{Gram}$-positive; $(-\mathrm{ve})=$ Gram-negative

Compounds which had greater zones of inhibition, i.e., analogs $6,11,13,16$, and 17 , were subjected to further analyses (determination of MIC, and MBC) to test their activity against other commonly occurring microbes. These results are presented in Tables 2 and 3 . According to Table 2, compound 6 exhibited the same activity irrespective of the tested organism. In contrast, compound 11 showed the highest MIC value (1.249 $\mathrm{mg} / \mathrm{mL}$ ) against $B$. subtilis and $P$. aeruginosa and the lowest $(0.524 \mathrm{mg} / \mathrm{mL})$ against $E$. coli. The MIC of compound 13 against $S$. abony was not determined, as 13 did not show any inhibition in the primary screening test (Table 2). However, its highest MIC value was against $B$. subtilis and its lowest against E. coli. A significantly high MIC value $(0.78 \mathrm{mg} / \mathrm{mL})$ was obtained for compound 16 against B. subtilis, E. coli, and $S$. abony, suggesting similar types of activity, while its MIC value was significantly higher against $S$. aureus and $P$. aeruginosa (Table 2). Moreover, although MIC against $B$. subtilis was not found for compound $\mathbf{1 7}$, it exhibited the highest MIC value $(2.708 \mathrm{mg} / \mathrm{mL})$ against $S$. abony and much lower but the same MIC value against all the other tested bacteria.

Table 2. MIC values for compounds 6, 11, 13, 16, and 17 against selected bacteria

\begin{tabular}{cccccc}
\hline \multirow{2}{*}{ Compd. } & \multicolumn{5}{c}{ MIC values $(\mathbf{m g} / \mathbf{m L})$} \\
\cline { 2 - 6 } & subtilis & aureus & coli & abony & aeruginosa \\
\hline $\mathbf{6}$ & 0.520 & 0.520 & 0.520 & 0.520 & 0.520 \\
$\mathbf{1 1}$ & 1.249 & 0.624 & 0.524 & 0.624 & 1.249 \\
$\mathbf{1 3}$ & 1.553 & 0.676 & 0.676 & $\mathrm{NF}$ & 1.353 \\
$\mathbf{1 6}$ & 0.780 & 2 & 0.780 & 0.780 & 2 \\
$\mathbf{1 7}$ & $\mathrm{NF}$ & 0.520 & 0.520 & 2.708 & 0.520 \\
\hline $\mathrm{NF}$
\end{tabular}

Furthermore, based on the MBC data in Table 3, compound 6 was equally active against all the bacteria with a MBC value of $2.083 \mathrm{mg} / \mathrm{mL}$. However, compound 11 exhibited a high MBC value against $B$. subtilis and $P$. aeruginosa, but a lower and unique MBC value against $S$. aureus, E. coli, and S. abony. Similar as before, no $\mathrm{MBC}$ was determined for compound 13 against $S$. abony, but significant $\mathrm{MBC}$ values were found for this compound against the other organisms. In the case of compound 16, a MBC value of up to 4 $\mathrm{mg} / \mathrm{mL}$ was found against $E$. coli and $P$. aeruginosa, while equivalent values were found against the other organisms. Moreover, compound $\mathbf{1 7}$ exhibited a significantly high $\mathrm{MBC}$ against $S$. abony $(5.416 \mathrm{mg} \mathrm{mL}$ ${ }^{1}$ ), but were inactive against $B$. subtilis and exhibited good and similar values against $S$. aureus, E. coli, and P. aeruginosa.

Table 3. MBC values for compounds $6,11,13,16$, and 17 against Gram-positive and Gram-negative bacteria

\begin{tabular}{cccccc}
\hline & \multicolumn{5}{c}{ MBC values $(\mathbf{m g} / \mathbf{m L})$} \\
\cline { 2 - 6 } Compd. & $\begin{array}{c}\text { B. } \\
\text { subtilis }\end{array}$ & $\begin{array}{c}\text { S. } \\
\text { aureus }\end{array}$ & $\begin{array}{c}\boldsymbol{E} \text { coli } \\
\text { cobony }\end{array}$ & $\begin{array}{c}\text { S. } \\
\text { aboruginosa }\end{array}$ \\
\hline $\mathbf{6}$ & 2.083 & 2.083 & 2.083 & 2.083 & 2.083 \\
$\mathbf{1 1}$ & 4.999 & 2.499 & 2.499 & 2.499 & 4.999 \\
$\mathbf{1 3}$ & 5.416 & 2.708 & 2.708 & $\mathrm{NF}$ & 5.416 \\
$\mathbf{1 6}$ & 3.124 & 4 & 3.124 & 3.124 & 4 \\
$\mathbf{1 7}$ & $\mathrm{NF}$ & 2.083 & 2.083 & 5.416 & 2.083 \\
\hline
\end{tabular}

$\mathrm{NF}=$ Not Found

\subsection{Determination of the antifungal activity of uridine derivatives}

The antifungal screening test indicated that compound 12 significantly inhibited the mycelial growth of both the A. niger $(75.56 \pm 1.28 \%)$ and $A$. flavus $(77.77 \pm 1.20 \%)$ fungi (Figure 2$)$, and the values were much higher than those of nystatin. However, the highest percentage of inhibition $(89.89 \pm 1.11 \%)$ was obtained for compound 11, while compound $\mathbf{3}$ exhibited the lowest inhibition against A. niger. Furthermore, compounds 9, 16, and 17 exhibited moderate inhibition 
against $A$. flavus, while no inhibition was observed for compounds $\mathbf{2}$ and $\mathbf{1 4}$ against both fungal pathogens.

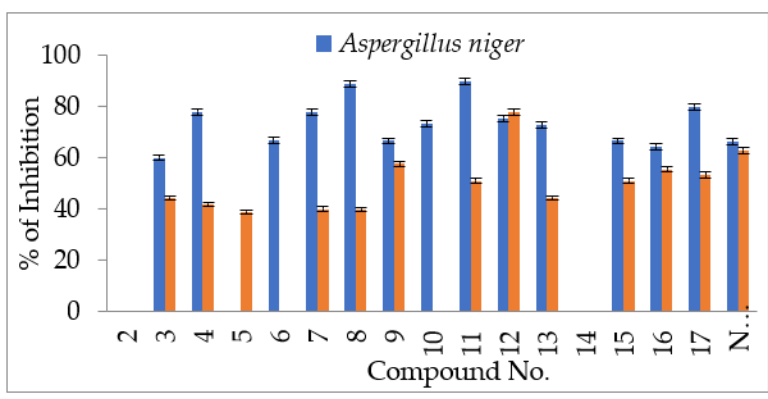

Figure 2. Antifungal activities graph of the test compounds

In summary, the synthesized compounds exhibited very good antimicrobial activity. Especially the presence of different acyl moieties, including the butyryl, octanoyl, lauroyl, trityl, and 3-bromobenzoyl groups, significantly improved the antimicrobial activity, being in line with our previous studies [43,44]. Therefore, taking into account the primary screening and the MIC and MBC data analysis results, compounds $6,13,16$, and 17 could be used as potential antifungal therapeutics after further investigation.

\subsection{SAR study}

SAR studies can be used to predict the biological activity of a pharmaceutical target from its molecular structure. This powerful technology is often used in drug discovery to guide the acquisition or synthesis of desired new compounds or characterize existing molecules. Given that the chemical structure of the compounds is interrelated for their biological activity, which has been widely examined in recent years $[45,46]$, an SAR study of the synthesized compounds (Scheme 1) was performed in this study as well, based on the experimental results. The introduction of the myristoyl group at the C-5' position and the butyryl group or the lauroyl group at the $\mathrm{C}-2^{\prime}$ and $\mathrm{C}^{-3}{ }^{\prime}$ positions in compounds 11 and 13, respectively (Figure 3), enhanced the antimicrobial activity of uridine (1). Compound $\mathbf{1 3}$ $(21 \pm 0.35)$ was found more active than compound $\mathbf{1 1}$ $(20 \pm 0.34)$ against same bacteria $P$. aeruginosa because lauroyl group provide higher hydrophobicity than butyryl group. Compound $16(21 \pm 0.35)$ showed same activity as Compound $\mathbf{1 3}$ due to incorporation of triphenylmethyl group (three aromatic ring) at C-2' and C-3' position and myristoyl group at $\mathrm{C}-5^{\prime}$ position. This result revealed that hydrophobicity modified the functionality and aromatic ring is also candidates for this credit though in some cases it may be matter of arbitrary.

Moreover, the introduction of the lauroyl and myristoyl groups gradually increased the hydrophobicity of the uridine derivatives. The hydrophobicity of a material is an essential parameter for its bioactivity, as it may affect the toxicity or alter the membrane integrity, because it is directly related to membrane permeation [43]. Hunt and Judge [30, 47] also suggested that the potency of aliphatic alcohols is directly related to their lipid solubility due to the hydrophobic interactions between the alkyl chains of the alcohol and the lipid membrane regions. Thus, it was assumed that similar hydrophobic interactions may develop between the acyl chains of the uridine derivatives that accumulate in the lipid-like bacterial membranes, leading to a significant loss of their membrane permeability, which ultimately leads to bacterial death $[30,47]$.

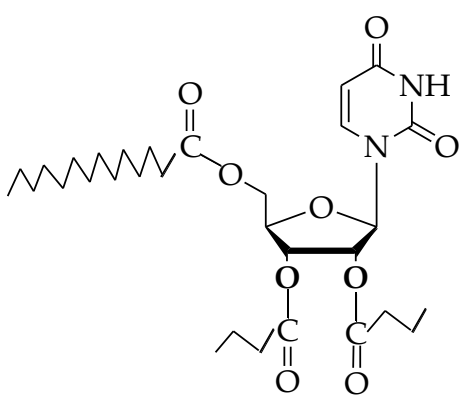

11

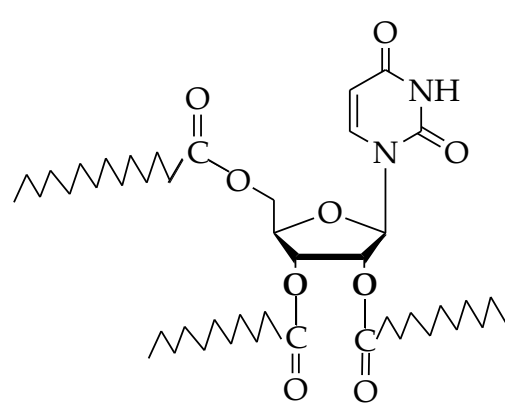

13

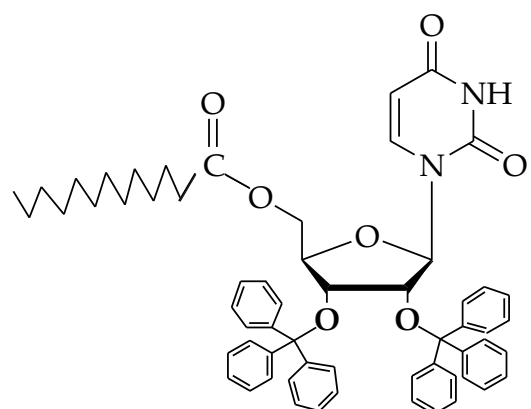

16

Figure 3. Chemical structure of uridine derivatives 11, 13, and 16

\subsection{Thermogravimetric analysis}

The thermal behavior of the synthesized compounds under optimized conditions was investigated via the TGA technique. The synthesized compounds showed a minor weight loss of about $5-10 \mathrm{wt} \%$ at a temperature lower than $105^{\circ} \mathrm{C}$, probably due to the evaporation of the trapped and absorbed water molecules. In addition, the maximum weight loss (about $41.5 \mathrm{wt}$. \%) was observed in the temperature range $105-200{ }^{\circ} \mathrm{C}$. An example of TGA curves is provided in Figure 4 for the uridine derivatives 6 and 17. The almost horizontal parts of the curves $(A B \& C D)$ indicated that there was no change in weight, while the declining part $\mathrm{BC}$ suggested that a considerable weight loss occurred. The weight of analogs $6,9,10,11,13,14,15,16$, and 17 remained constant up to temperatures of $85.67{ }^{\circ} \mathrm{C}, 77.57^{\circ} \mathrm{C}$, $100.86{ }^{\circ} \mathrm{C}, 102.20{ }^{\circ} \mathrm{C}, 78.08{ }^{\circ} \mathrm{C}, 105.46{ }^{\circ} \mathrm{C}, 151.72{ }^{\circ} \mathrm{C}$, $105.65{ }^{\circ} \mathrm{C}$, and $135.43^{\circ} \mathrm{C}$, respectively, indicating their thermal stability at these temperatures. However, at temperatures higher than those indicated, weight loss was observed, implying the beginning of decomposition. 


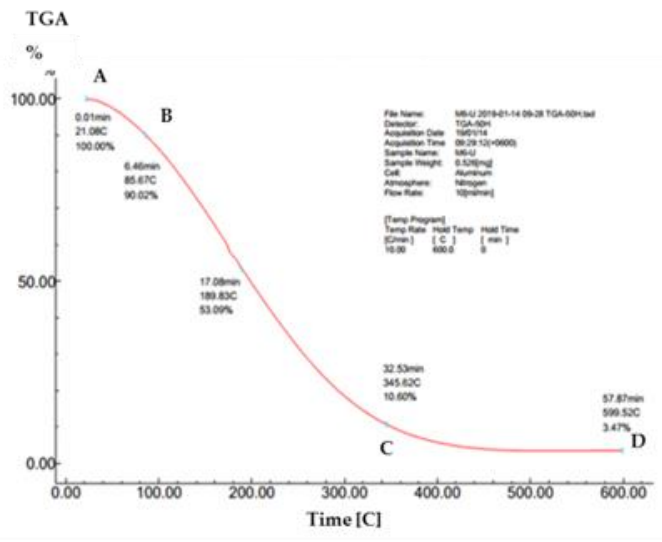

6

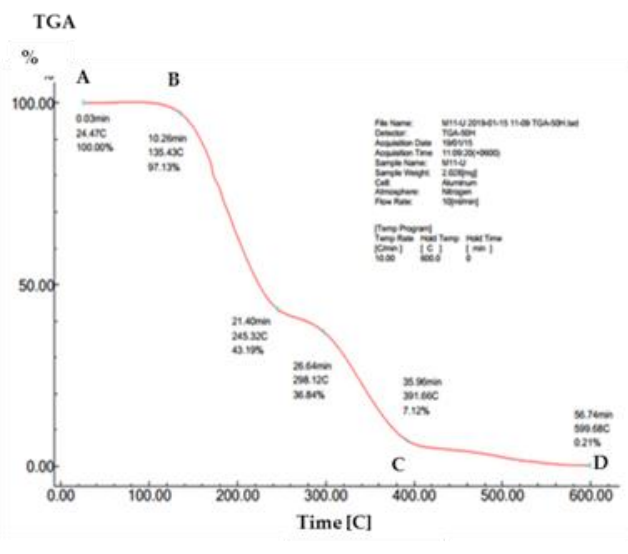

17

Figure 4. TGA curves of compounds $\mathbf{6}$ and $\mathbf{1 7}$

\subsection{XRD analysis}

The XRD patterns of the studied compounds were obtained according to the rules presented in Table 4 . The crystallographic structures of the synthesized compounds $6,9,10,11,13,14,15,16$, and 17 were evaluated by powder XRD (PXRD) at rt and for an example of XRD pattern is provided in Figure 5 for the derivatives of 6 and $\mathbf{1 6 .}$

Table 4. Rules for the determination of crystal lattice type

\begin{tabular}{cc}
\hline Lattice type & Rules reflecting to each lattice type \\
\hline Primitive, $\mathrm{P}$ & None \\
Body centered, I & $* \mathrm{hkl} ; \mathrm{h}+\mathrm{k}+\mathrm{l}=2 \mathrm{n}$ \\
Face centered, F & $\mathrm{hkl} ; \mathrm{h}, \mathrm{k}, \mathrm{l}$ either all odd or all even \\
Side centered, C & $\mathrm{hkl} ; \mathrm{h}+\mathrm{k}=2 \mathrm{n}$ \\
Rhombohedral & $\mathrm{hkl} ;(-\mathrm{h})+\mathrm{k}+\mathrm{l}=3 \mathrm{n}$ or $(\mathrm{h}-\mathrm{k})+\mathrm{l}=3 \mathrm{n}$ \\
\hline
\end{tabular}

$* \mathrm{~h}, \mathrm{k}$, and $\mathrm{l}$ are the Miller indices.

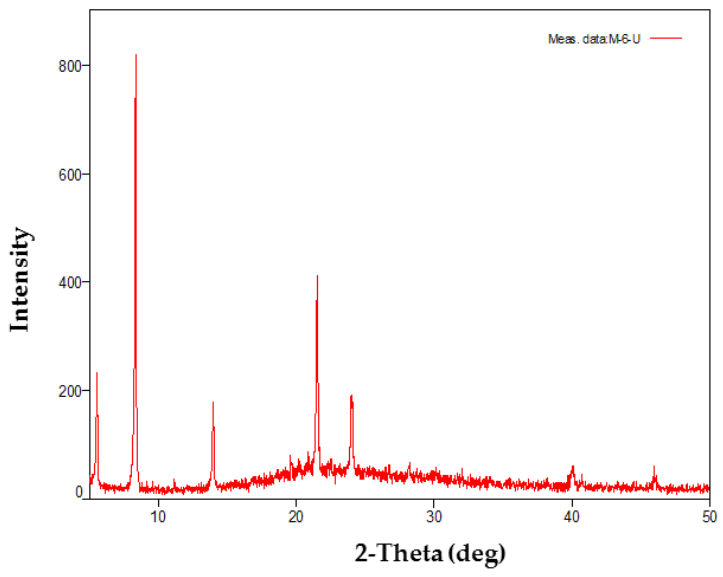

6
The XRD patterns of the pure compounds synthesized under optimized conditions were recorded in the $2 \theta$ range of $0^{\circ}-50^{\circ}$. More specifically, peaks at $8.344^{\circ}$ and $21.478^{\circ}$ (h, k, l: 110 and 330 ), $8.420^{\circ}$ and $21.544^{\circ}\left(\mathrm{h}, \mathrm{k}, \mathrm{l}: 110\right.$ and 222), $8.378^{\circ}$ and $21.503^{\circ}(\mathrm{h}, \mathrm{k}$, $\mathrm{l}: 110$ and 330$), 9.263^{\circ}$ and $21.415^{\circ}(\mathrm{h}, \mathrm{k}, \mathrm{l}: 110$ and 311 ), and $7.445^{\circ}$ and $21.511^{\circ}$ (h, k, l: 100 and 221) were detected for compounds, $6,9, \mathbf{1 0}, \mathbf{1 3}$, and 14, respectively, indicating the formation of typical phases. According to phase analysis, compounds synthesized under this method were highly pure, as no impurities were detected in the XRD patterns. Applying the rules listed in Table 4, the lattice structure for each of the synthesized uridine derivatives was determined. Compounds 6, 10, and 11 satisfied the rule $h+k+l=2 n$, which was related to a body-centered lattice (I), whereas analogs 9, 13, 14, 15, 16, and 17 were identified as primitive $(\mathrm{P})$ for which no specific rule applies.

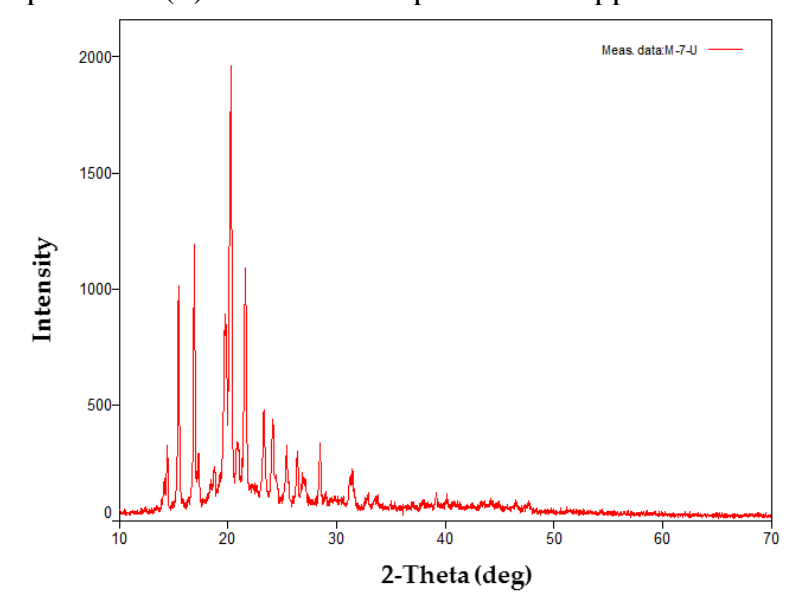

16

Figure 5. XRD patterns of compounds $\mathbf{6}$ and $\mathbf{1 6}$

\subsection{Thermodynamic analysis}

Changes in molecular structure significantly affect the structural properties including thermal and molecular orbital parameters. The spontaneity of a reaction and the stability of a product can be elucidated by the free energy and enthalpy values [48]. Highly negative values indicate higher thermal stability. In drug design, the hydrogen bond formation and non-bonded interactions are also affected by the dipole moment, where higher dipole moments can improve the binding properties [49]. Herein, the highest free energy (-9616.780 Hartree) was observed for the uridine derivative $\mathbf{8}$, and the highest dipole moment (7.597 Debye) was observed for the uridine derivative $\mathbf{1 1}$ (Table 5). 
Table 5. Stoichiometry, electronic energy, enthalpy, Gibbs free energy (in Hartree), and dipole moment (in Debye) of the synthesized uridine derivatives

\begin{tabular}{|c|c|c|c|c|c|}
\hline Compd. & Stoichiometry & $\begin{array}{c}\text { Electronic } \\
\text { Energy }\end{array}$ & Enthaly & $\begin{array}{c}\text { Gibbs free } \\
\text { Energy }\end{array}$ & Dipole moment \\
\hline 2 & $\mathrm{C}_{16} \mathrm{H}_{15} \mathrm{O}_{7} \mathrm{~N}_{2} \mathrm{Br}$ & -4103.952 & -4111.732 & -4002.002 & 5.020 \\
\hline 3 & $\mathrm{C}_{20} \mathrm{H}_{19} \mathrm{O}_{9} \mathrm{~N}_{2} \mathrm{Br}$ & -4113.976 & -4112.974 & -4113.068 & 6.270 \\
\hline 4 & $\mathrm{C}_{22} \mathrm{H}_{23} \mathrm{O}_{9} \mathrm{~N}_{2} \mathrm{Br}$ & -4191.121 & -4191.120 & -4191.222 & 5.196 \\
\hline 5 & $\mathrm{C}_{24} \mathrm{H}_{27} \mathrm{O}_{9} \mathrm{~N}_{2} \mathrm{Br}$ & -4269.250 & -4269.249 & -4269.357 & 7.462 \\
\hline 6 & $\mathrm{C}_{44} \mathrm{H}_{67} \mathrm{O}_{9} \mathrm{~N}_{2} \mathrm{Br}$ & -5050.690 & -5050.689 & -5050.853 & 4.713 \\
\hline 7 & $\mathrm{C}_{54} \mathrm{H}_{43} \mathrm{O}_{7} \mathrm{~N}_{2} \mathrm{Br}$ & -4753.120 & -4667.134 & -4332.234 & 4.412 \\
\hline 8 & $\mathrm{C}_{30} \mathrm{H}_{21} \mathrm{O}_{9} \mathrm{~N}_{2} \mathrm{Br}_{3}$ & -9616.663 & -9616.662 & -9616.780 & 4.368 \\
\hline 9 & $\mathrm{C}_{23} \mathrm{H}_{38} \mathrm{O}_{7} \mathrm{~N}_{2}$ & -2410.143 & -2511.176 & -2643.276 & 3.221 \\
\hline 10 & $\mathrm{C}_{27} \mathrm{H}_{42} \mathrm{O}_{9} \mathrm{~N}_{2}$ & -1829.974 & -1829.973 & -1830.088 & 6.471 \\
\hline 11 & $\mathrm{C}_{31} \mathrm{H}_{50} \mathrm{O}_{9} \mathrm{~N}_{2}$ & -1986.272 & -1986.271 & -1986.399 & 7.597 \\
\hline 12 & $\mathrm{C}_{39} \mathrm{H}_{66} \mathrm{O}_{9} \mathrm{~N}_{2}$ & -2298.809 & -2298.808 & -2298.972 & 5.032 \\
\hline 13 & $\mathrm{C}_{47} \mathrm{H}_{82} \mathrm{O}_{9} \mathrm{~N}_{2}$ & -2611.398 & -2611.397 & -2611.580 & 3.481 \\
\hline 14 & $\mathrm{C}_{55} \mathrm{H}_{98} \mathrm{O}_{9} \mathrm{~N}_{2}$ & -2832.214 & -2852.165 & -2833.089 & 5.439 \\
\hline 15 & $\mathrm{C}_{41} \mathrm{H}_{71} \mathrm{O}_{9} \mathrm{~N}_{2}$ & -2756.432 & -2732.786 & -2709.098 & 4.989 \\
\hline 16 & $\mathrm{C}_{61} \mathrm{H}_{66} \mathrm{O}_{7} \mathrm{~N}_{2}$ & -2982.654 & -2982.653 & -2982.807 & 6.217 \\
\hline 17 & $\mathrm{C}_{37} \mathrm{H}_{44} \mathrm{O}_{9} \mathrm{~N}_{2} \mathrm{Br}_{2}$ & -7333.655 & -7333.654 & -7333.799 & 5.305 \\
\hline
\end{tabular}

\subsection{Molecular orbital analysis}

The HOMO-LUMO energies (Hartree), HOMOLUMO gap, hardness ( $\eta$ ), and softness (S) of all uridine derivatives are outlined in Table 6 . The electronic absorption is related to the transition from the ground to the first excited state and is mainly described by one electron excitation from HOMO to LUMO [50]. The chemical hardness, softness, and chemical potential values depend on the HOMO-LUMO energy. Moreover, the kinetic stability increases with the increase in the HOMO-LUMO gap, leading to an increase in the energy required for the electron transfer from the ground HOMO state to the excited LUMO state. The HOMO-LUMO gap of $\mathbf{5}$ was determined at 0.29502 Hartree, but lower gaps were observed for more reactive uridine derivatives, among which 16 exhibited the lowest HOMO-LUMO gap (0.16568 Hartree) with the highest $S$ (12.07146), which might have contributed to its higher chemical reactivity and polarizability compared to other analogs.

Table 6. Energy (Hartree) gaps of HOMOs and LUMOs, HOMO-LUMO gap, $\eta$, and $S$ of the uridine derivatives

\begin{tabular}{|c|c|c|c|c|c|}
\hline Compd. & $\begin{array}{c}\text { EHOMO } \\
\text { (Hartree) }\end{array}$ & $\begin{array}{c}\text { ELUMO } \\
\text { (Hartree) }\end{array}$ & $\begin{array}{c}\text { HOMO-LUMO } \\
\text { gap (Hartree) }\end{array}$ & Hardness $(\eta)$ & Softness $(S)$ \\
\hline 2 & -0.23855 & -0.05773 & 0.17865 & 0.08765 & 9.86540 \\
\hline 3 & -0.23974 & -0.05992 & 0.17982 & 0.08991 & 11.12223 \\
\hline 4 & -0.24767 & -0.05004 & 0.19763 & 0.09882 & 10.11992 \\
\hline 5 & -0.24452 & -0.05050 & 0.29502 & 0.14751 & 6.77920 \\
\hline 6 & -0.25592 & -0.06350 & 0.19242 & 0.09621 & 10.39392 \\
\hline 7 & -0.24018 & -0.06154 & 0.18654 & 0.08765 & 10.65432 \\
\hline 8 & -0.25030 & -0.07171 & 0.17859 & 0.08929 & 11.19883 \\
\hline 9 & -0.23543 & -0.05021 & 0.19765 & 0.10432 & 9.61821 \\
\hline 10 & -0.24631 & -0.04042 & 0.20589 & 0.10295 & 9.71392 \\
\hline 11 & -0.23984 & -0.03235 & 0.20749 & 0.10375 & 9.63901 \\
\hline 12 & -0.23740 & -0.03008 & 0.20732 & 0.10366 & 9.64692 \\
\hline 13 & -0.23970 & -0.03517 & 0.20543 & 0.10227 & 9.77851 \\
\hline 14 & -0.22431 & -0.03069 & 0.20865 & 0.10198 & 9.76810 \\
\hline 15 & -0.21874 & -0.04864 & 0.20976 & 0.08131 & 9.94321 \\
\hline 16 & -0.21621 & -0.05053 & 0.16568 & 0.08284 & 12.07146 \\
\hline 17 & -0.23901 & -0.06734 & 0.17167 & 0.08583 & 11.65025 \\
\hline
\end{tabular}

\subsection{Interaction and binding affinity of uridine derivatives against 5 WS1}

Molecular docking studies were performed against the human gene protein 5WS1 (PDB ID: 5WS1, protein: poly ADP-ribose polymerase, gene: PARP1), which plays a key role in DNA repair. The binding affinities of the uridine derivatives to the protein are summarized in Table 5. Generally, higher negative binding affinity values indicate stronger binding between the drugs and the receptor protein, while the development of strong hydrogen bonds significantly contributes to the increase in the drug-receptor binding affinity. Moreover, the binding affinity and specialty change with the addition of different functional groups as substituents. The optimized structures of the uridine derivatives $\mathbf{6}, \mathbf{1 1}$, and 16 are depicted in Figure 6 and their docked structures with 5WS1 in Figure 7, where the multiple non-bonded interactions are clearly indicated. Among these derivatives, compound $\mathbf{1 1}$ exhibited the highest binding affinity. Its docked confirmation with the receptor protein 5WS1 and superimposed view of all compounds after rigid docking are illustrated in Figure 8. 


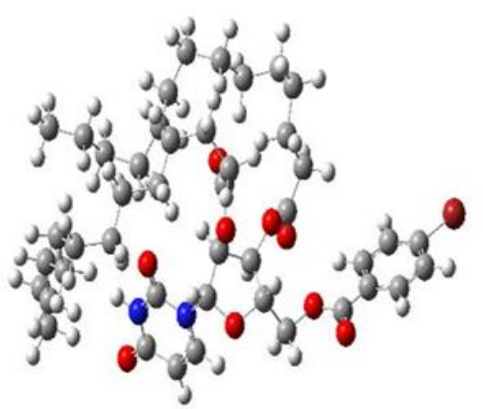

6

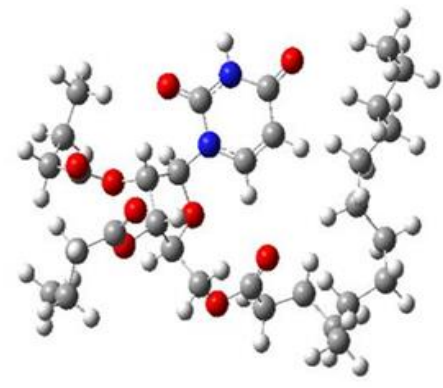

11

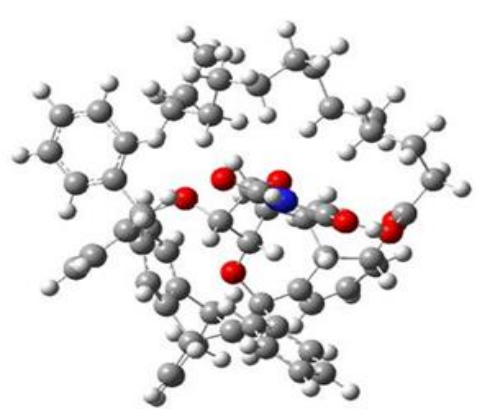

16

Figure 6. Optimized structures of the uridine derivatives 6, 11, and $\mathbf{1 6}$ calculated at the DFT/-B3LYP/-3-21G level

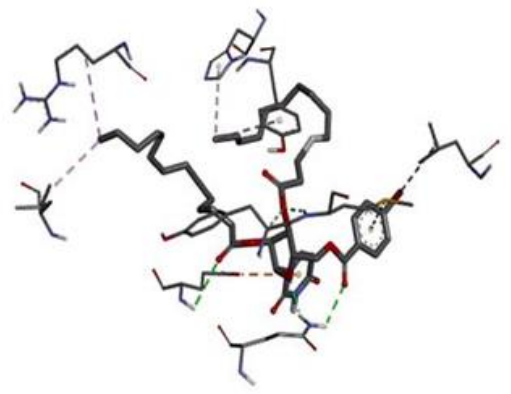

6

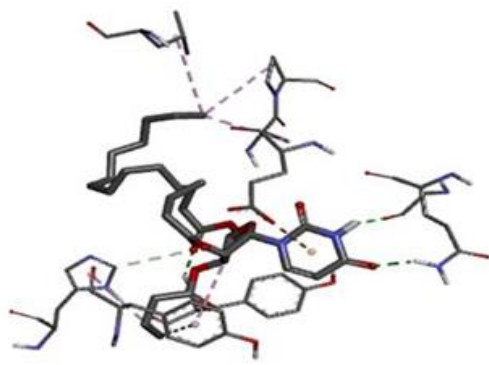

11

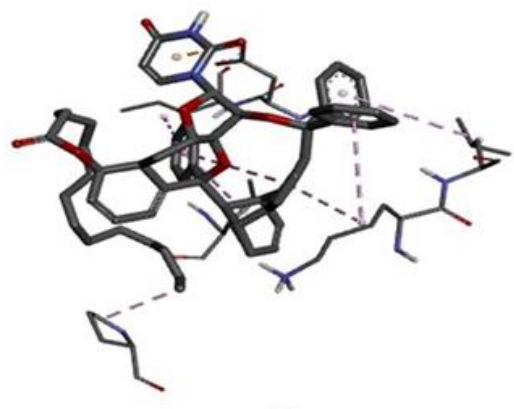

16

Figure 7. Binding interactions of the uridine derivatives $\mathbf{6 , 1 1}$, and $\mathbf{1 6}$ with the human gene protein 5WS1

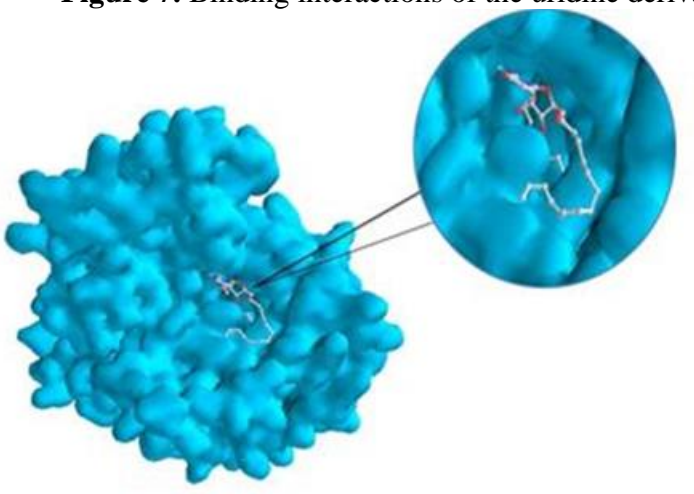

A

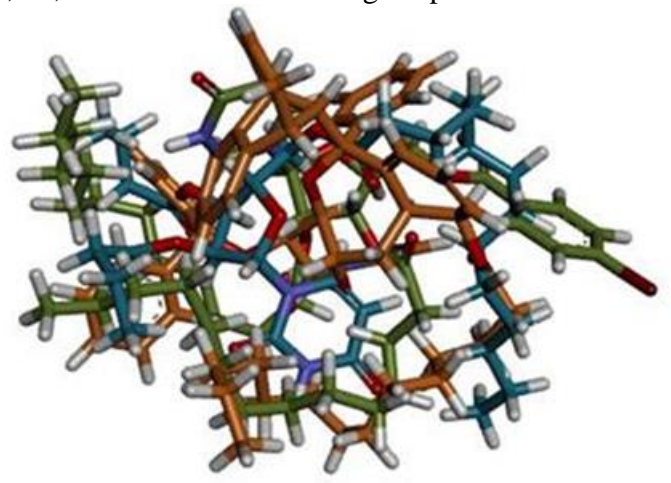

B

Figure 8. Docked conformation of compound $\mathbf{1 1}$ at inhibition bounding site of 5WS1 (A). Superimposed view of all compounds after rigid docking (B).

\subsection{MEP analysis}

The MEP is widely used as a reactivity map to identify the region that can more likely be attacked by electrophilic and nucleophilic charged moieties, such as reagents on organic molecules [51], allowing the interpretation of the biological recognition process and hydrogen bonding interactions. In addition, the MEP counter map provides a simple way to predict the interactions of various chemical structures and geometries. Determining MEP is particularly important, because it can simultaneously display the molecular size and shape, as well as the positive, negative, and neutral electrostatic potential regions using color grading, which are very useful for elucidating the relationship between a molecular structure and its physicochemical properties. The MEP of analogs 6, 11, and 16 was obtained based on the B3LYP level with the basis set 3$21 \mathrm{G}$ optimized result (Figure 9). In Figure 9, the potential increased in the order red < orange < yellow < green < blue, where the red color represents the maximum negative area, favorable site for electrophilic attacks, the blue color indicates the maximum positive area, favorable for nucleophilic attacks, and the green color represents the zero potential areas. 


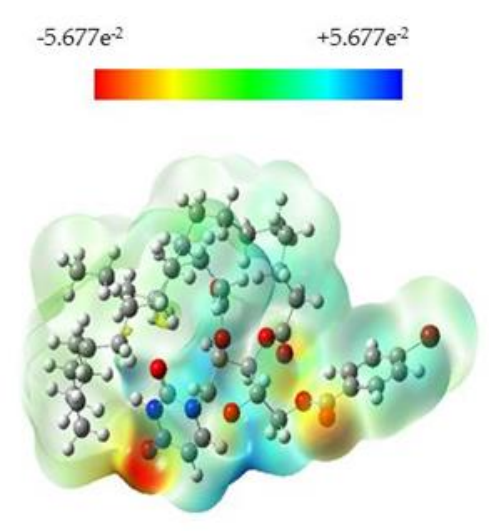

6

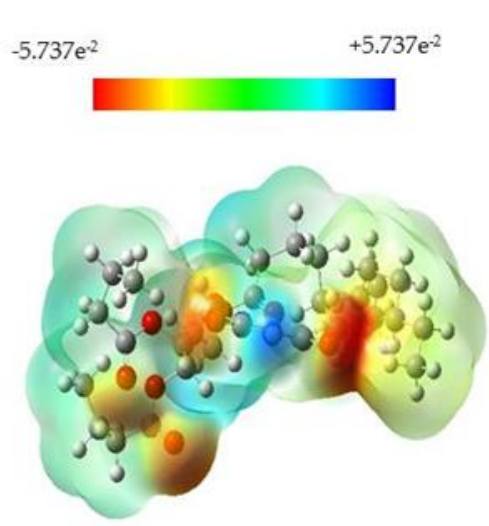

11

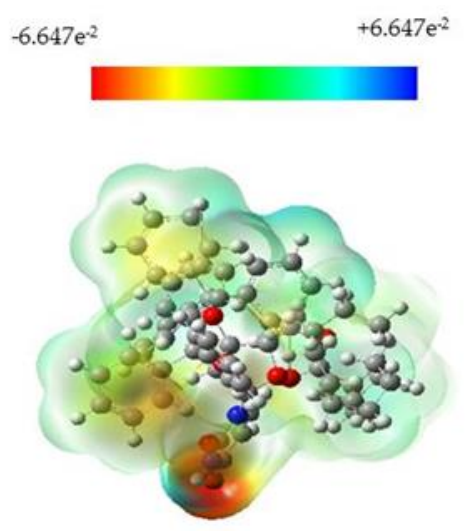

16

Figure 9. MEP map of the uridine derivatives $\mathbf{6}, \mathbf{1 1}$, and 16. The different values of electrostatic potential are indicated with different colors.

\subsection{SwissADME prediction}

The physicochemical properties of compounds $\mathbf{6}, \mathbf{1 1}$, and 16, such as their molar refractivity, total surface area (TPSA), and fraction $\mathrm{Cs} p^{3}$, were calculated from the
SwissADME online server. Analog 16 showed the highest molar refractivity, whereas compound $\mathbf{1 1}$ exhibited the highest total surface area and fraction $\mathrm{C} s p^{3}$ (Table 7).

Table 7. Binding affinity, polarizability, and selected physicochemical parameters of compounds $\mathbf{6}, \mathbf{1 1}$, and $\mathbf{1 6}$

\begin{tabular}{|c|c|c|c|c|c|c|}
\hline Compd. & $\begin{array}{c}\text { Protein } \\
\text { (PDB ID) }\end{array}$ & $\begin{array}{c}\text { Binding } \\
\text { affinity } \\
\text { (kcal/mol) }\end{array}$ & $\begin{array}{c}\text { Polarizability } \\
\text { (a.u) }\end{array}$ & $\begin{array}{l}\text { Molar } \\
\text { refractivity }\end{array}$ & $\begin{array}{l}\text { Total polar } \\
\text { surface area } \\
(\text { TPSA })(\AA)\end{array}$ & $\begin{array}{c}\text { Fraction } \\
\operatorname{Csp}^{3}\end{array}$ \\
\hline 6 & 5WS1 & -7.4 & 491.99666 & 228.21 & 108.85 & 0.67 \\
\hline 11 & 5WS1 & -7.8 & 346.19300 & 160.39 & 142.99 & 0.77 \\
\hline 16 & 5WS1 & -7.4 & 600.42333 & 277.84 & 108.85 & 0.33 \\
\hline
\end{tabular}

\subsection{ADMET prediction (pharmacokinetic properties)}

The pharmacokinetic properties (Table 8) of the synthesized uridine derivatives were also calculated to compare their absorption, metabolism, and toxicity. The admetSAR software [40] was used to clarify whether these uridine derivatives are non-carcinogenic and have oral toxicity in category III, so that they can be considered harmless for oral administration. As observed in Table 13, none of the prepared compounds inhibited the P-glycoprotein, something that would instead interrupt their efficient absorption, permeability, and retention, while all of them could cross the bloodbrain barrier (BBB). However, all the uridine derivatives were weak inhibitors of the human ether-à-go-go-related gene (hERG). Therefore, considering that the effective inhibition of hERG can lead to long QT syndrome [52], further investigation is required in this regard.

Table 8. Pharmacokinetic properties of selected uridine derivatives

\begin{tabular}{ccccccc}
\hline Compd. & BBB & $\begin{array}{c}\text { Human } \\
\text { intestinal } \\
\text { absorption }\end{array}$ & $\begin{array}{c}\text { P-glycoprotein } \\
\text { inhibitor }\end{array}$ & hERG & Carcinogen & $\begin{array}{c}\text { Acute } \\
\text { oral } \\
\text { toxicity }\end{array}$ \\
\hline $\mathbf{2}$ & $+(0.9782)$ & $+(0.8521)$ & $\mathrm{NI}(0.8931)$ & $\mathrm{WI}(0.7587)$ & $\mathrm{NC}(0.8567)$ & $\mathrm{III}$ \\
$\mathbf{3}$ & $+(0.9788)$ & $+(0.8545)$ & $\mathrm{NI}(0.8373)$ & $\mathrm{WI}(0.7434)$ & $\mathrm{NC}(0.8429)$ & III \\
$\mathbf{4}$ & $+(0.9788)$ & $+(0.8574)$ & $\mathrm{NI}(0.9049)$ & $\mathrm{WI}(0.8322)$ & $\mathrm{NC}(0.8571)$ & III \\
$\mathbf{5}$ & $+(0.9801)$ & $+(0.8574)$ & $\mathrm{NI}(0.8776)$ & $\mathrm{WI}(0.7545)$ & $\mathrm{NC}(0.8571)$ & III \\
$\mathbf{6}$ & $+(0.9790)$ & $+(0.8574)$ & $\mathrm{NI}(0.7721)$ & $\mathrm{WI}(0.6916)$ & $\mathrm{NC}(0.8571)$ & III \\
$\mathbf{7}$ & $+(0.9821)$ & $+(0.8562)$ & $\mathrm{NI}(0.8054)$ & $\mathrm{WI}(0.8103)$ & $\mathrm{NC}(0.8438)$ & III \\
$\mathbf{8}$ & $+(0.9808)$ & $+(0.8545)$ & $\mathrm{NI}(0.8122)$ & $\mathrm{WI}(0.8026)$ & $\mathrm{NC}(0.8000)$ & III \\
$\mathbf{9}$ & $+(0.9802)$ & $+(0.8767)$ & $\mathrm{NI}(0.7521)$ & $\mathrm{WI}(0.6541)$ & $\mathrm{NC}(0.8310)$ & III \\
$\mathbf{1 0}$ & $+(0.9821)$ & $+(0.8745)$ & $\mathrm{NI}(0.7597)$ & $\mathrm{WI}(0.7771)$ & $\mathrm{NC}(0.8143)$ & III \\
$\mathbf{1 1}$ & $+(0.9821)$ & $+(0.8745)$ & $\mathrm{NI}(0.7572)$ & $\mathrm{WI}(0.4716)$ & $\mathrm{NC}(0.8143)$ & $\mathrm{III}$ \\
$\mathbf{1 2}$ & $+(0.9821)$ & $+(0.8745)$ & $\mathrm{NI}(0.7495)$ & $\mathrm{WI}(0.3787)$ & $\mathrm{NC}(0.8143)$ & III \\
$\mathbf{1 3}$ & $+(0.9821)$ & $+(0.8745)$ & $\mathrm{NI}(0.7496)$ & $\mathrm{WI}(0.4311)$ & $\mathrm{NC}(0.8143)$ & III \\
$\mathbf{1 4}$ & $+(0.9811)$ & $+(0.8731)$ & $\mathrm{NI}(0.7555)$ & $\mathrm{WI}(0.6210)$ & $\mathrm{NC}(0.8106)$ & III \\
$\mathbf{1 5}$ & $+(0.9817)$ & $+(0.8750)$ & $\mathrm{NI}(0.8043)$ & $\mathrm{WI}(0.8311)$ & $\mathrm{NC}(0.8211)$ & III \\
$\mathbf{1 6}$ & $+(0.9834)$ & $+(0.8728)$ & $\mathrm{NI}(0.8155)$ & $\mathrm{WI}(0.8602)$ & $\mathrm{NC}(0.8286)$ & III \\
$\mathbf{1 7}$ & $+(0.9797)$ & $+(0.8574)$ & $\mathrm{NI}(0.7833)$ & $\mathrm{WI}(0.8590)$ & $\mathrm{NC}(0.8571)$ & III \\
\hline
\end{tabular}

Only a few computational and theoretical studies on uridine derivatives have been reported so far. The results of the present investigation showed that some of the currently synthesized acylated uridine derivatives could be additionally tested on a wide range of phytopathogenic fungi and bacteria before being sent to 
pesticide producing companies for further tests. The acylated derivatives of uridine (2-17) are also expected to be potential antiviral, antituberculosis, and antiinflammatory agents, suggesting the importance of additional studies in the future.

\section{Conclusions}

In conclusion, a feasible and efficient direct method was reported for the synthesis of uridine derivatives with excellent yields and short reaction times. The structure and purity of all the compounds were indicated by their spectral and physical data. Moreover, the acylation reactions were found to be very promising, as a single mono-substitution product was isolated by all the reactions in quite high yields. The antimicrobial results indicated that some of the new uridine derivatives may possess a wide range of antimicrobial activities. Therefore, 2-17 may be considered as a potential starting point for developing novel, improved antimicrobial agents against pathogenic organisms. Thus, the inherent stability and biochemical interactions of the uridine derivatives were also examined. Modified uridine derivatives had a lower HOMO-LUMO gap and significant pharmacokinetic properties. Especially analogs 6, 11, and 16 exhibited better binding affinities to the receptor protein 5WS1, while analog $\mathbf{6}$ was thermally more stable than $\mathbf{1 1}$ and $\mathbf{1 6}$. In addition, XRD studies revealed the basic structure of the novel analogs in nature. Consequently, this study may be useful for identifying the chemical, thermal, physicochemical, and binding properties of additional uridine derivatives, which could contribute to future developments in the field of pesticides and pharmaceuticals.

\section{Acknowledgements}

The authors are grateful to the Ministry of Science and Technology (MOST), Bangladesh for providing financial support (39.00.0000.09.14.009.2019/Phy's32/502, 2018-2019). This study was also supported by JSPS KAKENHI under grant No. JP19K06239 (Y.O. and Y.F.), and by the Research Promotion Fund of Yokohama City University, and Nagasaki International University. The authors are indebted to the Director, WMSRC, JU, Dhaka, Bangladesh for providing the spectra.

\section{Conflict of interest}

The authors declare no conflict of interest regarding the publication of this article.

\section{References}

[1] H. Nakata, K. Yoshioka, T. Kamiya, H. Tsuga, K. Oyanagi, Functions of heteromeric association between adenosine and P2Y receptors, Journal of Molecular Neuroscience 26 (2005) 233-238. https://doi.org/10.1385/JMN:26:2-3:233

[2] V.L. Damaraju, S. Damaraju, J.D. Young, S.A. Baldwin, J. Mackey, M.B. Sawyer, C.E. Cass, Nucleoside anticancer drugs: The role of nucleoside transporters in resistance to cancer chemotherapy, Oncogene 22 (2003) 7524-7536. https://doi.org/10.1038/sj.onc.1206952

[3] M.K. Kukhanova, Anti-HIV nucleoside drugs: A retrospective view into the future, Molecular Biology 46 (2012) 768-779. https://pubmed.ncbi.nlm.nih.gov/23350232/

[4] E.D. Clercq, G. Li, Approved antiviral drugs over the past 50 years, Clinical Microbiology Reviewes 29 (2016) 695-747.

https://doi.org/10.1128/CMR.00102-15

[5] E.D. Clercq, Antiviral drugs in current clinical use, Journal of Clinical Virology 30 (2004) 115-133. https://doi.org/10.1016/j.jcv.2004.02.009

[6] L.P. Jordheim, D. Durantel, F. Zoulim, C. Dumontet, Advances in the development of nucleoside and nucleotide analogues for cancer and viral diseases, Nature Reviews Drug Discovery 12 (2013) 447-464. https://doi.org/10.1038/nrd4010

[7] K. Juraj, T. Michal, P. Radek, H. Jan, D. Petr, H. Marián, H. Michal, Sugar modified pyrimido[4,5$b$ ]indole nucleosides: synthesis and antiviral activity, Medicinal Chemistry Communications 8 (2017) 1856-1862. https://doi.org/10.1039/c7md00319f

[8] L.K. Grove, X. Guo, S.H. Liu, Z. Gao, C.K. Chu, Y.C. Cheng, Anticancer activity of beta-Ldioxolane-cytidine, a novel nucleoside analogue with the unnatural L configuration, Comparative Study 55 (1995) 3008-3011. https://pubmed.ncbi.nlm.nih.gov/7606719/

[9] A.M. Mohamed, H.R. Al-Qalawi, W.A. El-Sayed, W.A. Arafa, M.S. Alhumaimess, A.K. Hassan, Anticancer activity of newly synthesized triazolopyridine derivatives and their nucleoside analogs, Acta Poloniae Pharmaceutica 72 (2015) 307-318. https://pubmed.ncbi.nlm.nih.gov/26642681/

[10] L. Vijaya, S.D. Damaraju, D.Y. James, A.B. Stephen, M. John, B.S. Michael, E.C. Carol, Nucleoside anticancer drugs: the role of nucleoside transporters in resistance to cancer chemotherapy, Oncogene 22 (2003) 7524-7536. https://doi.org/10.1038/sj.onc.1206952

[11] S. Rachakonda, L. Cartee, Challenges in antimicrobial drug discovery and the potential of nucleoside antibiotics, Current Medicinal Chemistry 11 (2004) 775-793. https://doi.org/10.2174/0929867043455774

[12] G. Ronquist, F. Niklasson, Uridine, xanthine, and urate contents in human seminal plasma, Archives of Andrology 13 (1984) 63-70. https://doi.org/10.3109/01485018408987501

[13] M. Itoh, D. Hagiwara, J. Notani, A simple and mild esterification method for carboxylic acids using sulfonate-type coupling reagents, Synthesis 1975 (1975) 456-458. https://doi.org/10.1055/s-197523804

[14] Y. Tsuda, E. Haque, Regioselective introduction of $p$-coumaroyl group to $\alpha$-L-arabino-pyranosides. Total synthesis of inundoside- $\mathrm{G}$ and inundosideD1, Chemical and Pharmaceutical Bulletin 31 (1983) 1437-1439. 
https://doi.org/10.1248/cpb.31.1437

[15] C. Andary, R. Wylde, C. Laffite, G. Privat, I. Winternitz, Structures of verbascoside and orobanchoside caffeic acid sugar esters from Orobanche rapumgenistae, Phytochemistry 21 (1982) 1123-1127. https://doi.org/10.1016/S00319422(00)82429-2

[16] H. Ishii, M. Nakamura, S. Seo, K. Tori, T. Tozoyo, Y. Yoshimura, Isolation, characterization and nuclear magnetic spectra of new saponins from the roots of Bupleurum falcatum L, Chemical and Pharmaceutical Bulletin 28 (1980) 2367-2373. https://doi.org/10.1248/cpb.28.2367

[17] A.K.M.S. Kabir, P. Dutta, M. N. Anwar, Synthesis of some new derivatives of D-mannose, Chittagong University Journal of Science 29 (2005) 1-8.

[18] A.F. El-Farargy, A.G. Amira, Synthesis of some purine nucleoside derivatives with expected biological activity, Current Organic Chemistry 13 (2009) 1842-1847. https://doi.org/10.2174/138527209789630488

[19] Z. Li, S. Chen, N. Jiang, G. Cui, Synthesis of triazole nucleoside derivatives, Nucleoside Nucleotide Nucleic Acids 22 (2003) 419-435. https://doi.org/10.1081/NCN-120022032

[20] S. K. Nermin, The synthesis of ribose and nucleoside derivatives, Madridge Journal of Novel Drug Research 2 (2018) 37-56. https://doi.org/10.18689/mjndr-1000107

[21] M.M. Ghorab, Z.H. Ismail, S.M.A. Gaward, A.A. Aziem, Antimicrobial activity of amino acid, imidazole and sulfonamide derivatives of pyrazolo[3,4-d]pyrimidine, Heteroatom Chemistry 15 (2004) 57-62. https://doi.org/10.1002/hc.10212

[22] R. Gupta, S. Paul, A.K. Gupta, P.L. Kachroo, S. Bani, Synthesis and biological activities of some substituted phenyl-3-(3-alkyl/aryl-5,6-dihydro-striazolo[3,4-b][1,3,4]thiazol-6-yl) indoles, Indian Journal of Chemistry 36 (1997) 707-710.

[23] S.M.A. Kawsar, M.O. Faruk, M.S. Rahman, Y. Fujii, Y. Ozeki, Regioselective synthesis, characterization and antimicrobial activities of some new monosaccharide derivatives, Sciencia Pharmaceutica 82 (2014) 1-20.

https://doi.org/10.3797/scipharm.1308-03

[24] S M.A. Kawsar, A.A. Hamida, A.U. Sheikh, M.K. Hossain, A.C. Shagir, A.F.M. Sanaullah, M.A. Manchur, H. Imtiaj, Y. Ogawa, Y. Fujii, Y. Koide, Y. Ozeki, Chemically modified uridine molecules incorporating acyl residues to enhance antibacterial and cytotoxic activities, International Journal of Organic Chemistry 5 (2015) 232-245. https://doi.org/10.4236/ijoc.2015.54023

[25] M. Uzzaman, M.N. Uddin, Optimization of structures, biochemical properties of ketorolac and its degradation products based on computational studies, DARU Journal of Pharmaceutical Science 27 (2019) 71-82. https://doi.org/10.1007/s40199019-00243-w

[26] M. Uzzaman and M. J. Hoque, Physiochemical, molecular docking, and pharmacokinetic studies of Naproxen and modified derivatives based on DFT,
International Journal of Scientific Research and Management 6 (2018) C-2018-12-19.

https://doi.org/10.18535/ijsrm/v6i9.c01

[27] A.W. Bauer, W.M.M. Kirby, J.C. Sherris, M. Turck, Antibiotic susceptibility testing by a standardized single disk method, American Journal of Clinical Pathology 45 (1966) 493-496. https://pubmed.ncbi.nlm.nih.gov/5325707/

[28] D. Msterdam, Susceptibility testing of antimicrobials in liquid media, In Lorian, V., (ed.), Antibiotics in laboratory medicine, $5^{\text {th }}$ ed. Williams, L., Wilkins, Philadelphia, PA, 2005 61, ISBN 0-7817-4983-2.

[29] R.K. Grover, J.D. Moore, Toximetric studies of fungicides against the brown rot organisms, Sclerotinia fructicola and S. laxa, Phytopathology 52 (1962) 876-879.

[30] W.A. Hunt, The effects of aliphatic alcohols on the biophysical and biochemical correlates of membrane function, Advances in Experimental Medicine and Biology 56 (1975) 195-210. https://doi.org/10.1007/978-1-4684-7529-6_9

[31] Y.M. Kim, S. Farrah, R.H. Baney, Structureantimicrobial activity relationship for silanols, a new class of disinfectants, compared with alcohols and phenols, International Journal of Antimicrobial Agents 29 (2007) 217-222. https://doi.org/10.1016/j.ijantimicag.2006.08.036

[32] R.A. Gaussian M.J. Frisch, G.W. Trucks, H.B. Schlegel, G.E. Scuseria, M.A. Robb, J.R. Cheeseman, G. Scalmani, V. Barone, B. Mennucci, G.A. Petersson, et al., Gaussian, Inc., Wallingford CT. 2009

[33] A.D. Becke, Density-functional exchange-energy approximation with correct asymptotic behaviour, Physical Review A 38 (1988) 3098-3100. https://doi.org/10.1103/physreva.38.3098

[34] C. Lee, W. Yang, R.G. Parr, Development of the colle-Salvetti correlation-energy formula into a functional of the electron density, Physical Reviews B 37 (1988) 785-789. https://doi.org/10.1103/physrevb.37.785

[35] R. G. Pearson, Absolute electronegativity and hardness correlated with molecular orbital theory, Proceedings of the National Academy of Sciences 83 (1986) 8440-8441. https://doi.org/10.1073/pnas.83.22.8440

[36] M.J. Lucido, B.J. Orlando, A.J. Vecchio, M.G. Malkowski, Crystal structure of aspirin-acylated human cyclooxygenase-2: Insight into the formation of products with reversed stereochemistry, Biochemistry 55 (2016) 12261238. https://doi.org/10.1021/acs.biochem.5b01378

[37] W.L. Delano, The PyMOL molecular graphics system. de-lano scientific, San Carlos, CA, USA, 2002. http://wwwpymolorg

[38] N. Guex, M.C. Peitsch, SWISS-MODEL and the Swiss-Pdb Viewer: An environment for comparative protein modeling, Electrophoresis 18 (1997) 2714-2723. https://doi.org/10.1002/elps.1150181505 
[39] S. Dallakyan, A.J. Olson, Small-molecule library screening by docking with PyRx. In: J.E. Hempel, C.H. Williams, C.C. Hong, (eds.) Chemical biology: methods and protocols. Springer, New York, NY, 2015, 243-250. https://doi.org/10.1007/978-1-4939-2269-7_19

[40] F. Cheng, W. Li, Y. Zhou, J. Shen, Z. Wu, G. Liu, admetSAR: A comprehensive source and free tool for assessment of chemical ADMET properties, Journal of Chemical Information and Modeling 52 (2012) 3099-3105. https://doi.org/10.1021/ci300367a

[41] S.R. Devi, S. Jesmin, M. Rahman, M.A. Manchur, Y. Fujii, R.A. Kanaly, Y. Ozeki, S.M.A. Kawsar, Microbial efficacy and two step synthesis of uridine derivatives with spectral characterization, ACTA Pharmaceutica Sciencia 57 (2019) 47-68. https://doi.org/10.23893/1307-2080.APS.05704

[42] S.M.A. Kawsar, M. Islam, S. Jesmin, M.A. Manchur, I. Hasan, S. Rajia, Evaluation of the antimicrobial activity and cytotoxic effect of some uridine derivatives, International Journal of Biosciences 12 (2018) 211-219. http://dx.doi.org/10.12692/ijb/12.6.211-219

[43] M. Arifuzzaman, M.M. Islam, M.M. Rahman, A.R. Mohammad, S.M.A. Kawsar, An efficient approach to the synthesis of thymidine derivatives containing various acyl groups: characterization and antibacterial activities, ACTA Pharmaceutica Sciencia 56 (2018) 7-22. https://doi.org/10.23893/1307-2080.APS.05622

[44] M.M.H. Misbah, J. Ferdous, M.Z.H. Bulbul, T.S. Chowdhury, S. Dey, I. Hasan, S.M.A. Kawsar, Evaluation of MIC, MBC, MFC and anticancer activities of acylated methyl $\beta$-Dgalactopyranoside esters, International Journal of Biosciences 16 (2020) 299-309. http://dx.doi.org/10.12692/ijb/16.4.299-309

[45] C. Huseyin, S. Murat, G. Murat, D. Serdar, K. Gulru, T.S. Claudiu, E. Deniz, Inhibition of acetylcholinesterase and butyrylcholinesterase with uracil derivatives: kinetic and computational studies, Journal of Enzyme Inhibition and Medicinal Chemistry 34 (2019) 429-437. https://doi.org/10.1080/14756366.2018.1543288

[46] S. Kumaresan, V. Senthilkumar, A. Stephen, B.S. Balakumar, GC-MS analysis and pass-assisted prediction of biological activity spectra of extract of Phomopsis sp. isolated from Andrographis paniculata, World Journal of Pharmaceutical Research 4 (2015) 1035-1053. https://wjpr.net/dashboard/abstract_id/1994

[47] V. Judge, B. Narasimhan, M. Ahuja, D. Sriram, P. Yogeeswari, E.D. Clercq, C. Pannecouque, J. Balzarini, Synthesis, antimycobacterial, antiviral, antimicrobial activity and QSAR studies of N(2)acyl isonicotinic acid hydrazide derivatives, Medicinal Chemistry 9 (2013) 53-76. https://doi.org/10.2174/157340613804488404

[48] N. Cohen, S. W. Benson, Estimation of heats of formation of organic compounds by additivity methods, Chemical Reviews 93 (1993) 24192438. https://doi.org/10.1021/cr00023a005

[49] E.J. Lien, Z.R. Guo, R.L. Li, C.T. Su, Use of dipole moment as a parameter in drug-receptor interaction and quantitative structure-activity relationship studies, Journal of Pharmaceutical Sciences 71 (1982) 641-655 https://doi.org/10.1002/jps.2600710611

[50] S. Saravanan, V. Balachandran, Quantum chemical studies, natural bond orbital analysis and thermodynamic function of 2,5-dichlorophenylisocyanate, Spectrochimica Acta Part A: Moecular and Biomolecular Spectroscopy 120 (2014) 351-364 https://doi.org/10.1016/j.saa.2013.10.042

[51] M.L. Amin, P-glycoprotein inhibition for optimal drug delivery, Drug Target Insight 7 (2013) 27-34. https://doi.org/10.4137/DTI.S12519

[52] C. Sanguinetti, M.T. Firouz, hERG potassium channels and cardiac arrhythmia, Nature 440 (2006) 463-469. https://doi.org/10.1038/nature04710

Received: 05.12.2020

Received in revised form: 23.01.2021 Accepted: 24.01.2021 